\title{
High-resolution spectroscopic follow-up of OGLE planetary transit candidates in the Galactic bulge: two possible Jupiter-mass planets and two blends
}

\author{
Maciej Konacki ${ }^{1}$ \\ Department of Geological and Planetary Sciences, California Institute of Technology, MS \\ 150-21, Pasadena, CA 91125, USA \\ Nicolaus Copernicus Astronomical Center, Polish Academy of Sciences, Rabianska 8, \\ 87-100 Toruń, Poland \\ Guillermo Torres², Dimitar D. Sasselov ${ }^{3}$ \\ Harvard-Smithsonian Center for Astrophysics, 60 Garden St., Cambridge, MA 02138, USA \\ Saurabh Jha ${ }^{4}$ \\ Department of Astronomy, University of California, Berkeley, CA 94720, USA \\ Harvard-Smithsonian Center for Astrophysics, 60 Garden St., Cambridge, MA 02138, USA
}

\begin{abstract}
We report the results of our campaign to follow-up spectroscopically several candidate extrasolar transiting planets from the OGLE-III survey in the direction of the Galactic center, announced in 2001 (Udalski et al. 2002a,b). All of these objects present shallow and periodic dips in brightness that may be due to planetary companions. Our high-resolution Keck I/HIRES observations have revealed two interesting cases (OGLE-TR-10 with a period of 3.1 days, and OGLE-TR-58 with a period of 4.3 days) that show no radial velocity variations at the level of $100-200 \mathrm{~m} \mathrm{~s}^{-1}$. If orbited by companions, their masses would be similar to Jupiter. With the information in hand (including the light curves) we are not able to rule out that these candidates are instead the result of contamination
\end{abstract}

\footnotetext{
${ }^{1}$ e-mail: maciej@gps.caltech.edu

${ }^{2}$ e-mail: gtorres@cfa.harvard.edu

${ }^{3}$ e-mail: dsasselov@cfa.harvard.edu

${ }^{4}$ e-mail: saurabh@astron.berkeley.edu
} 
from an eclipsing binary in the same line of sight (a "blend"). We discuss also the case of OGLE-TR-56 that was recently reported by Konacki et al. (2003) to have a Jupiter-size companion, based on an earlier analysis of our data, and we present supporting information. Two other candidates, OGLE-TR-3 and OGLETR-33, show clear evidence that they are blends. We describe tests carried out to characterize the stability of the HIRES spectrograph and its impact on the determination of precise velocities for faint stars ( $V \geq 15$ mag) using exposures of a Thorium-Argon lamp as the wavelength reference. Systematic effects are at the level of $100 \mathrm{~m} \mathrm{~s}^{-1}$ or smaller, and tend to dominate the total error budget. We also evaluate the precision attainable using the iodine gas absorption cell as an alternative fiducial, and we propose a simplified version of the standard procedure employed for high-precision Doppler planet searches that is very promising. Results from both this method and the classical Th-Ar technique show the feasibility of spectroscopic follow-up for faint targets in the range $V=14-17$.

Subject headings: techniques: radial velocities — binaries: eclipsing — stars: low-mass, brown dwarfs - planetary systems

\section{Introduction}

The application of high-precision radial velocity and pulsar timing techniques over the past decade has resulted in the discovery of more than 100 extrasolar giant planet candidates around solar-type stars (Schneider 2003), and also smaller Earth-mass planets around a millisecond pulsar (Konacki \& Wolszczan 2003; Wolszczan \& Frail 1992). With the photometric confirmation in 1999 of the giant planet around HD 209458 through the detection of its transits (Henry et al. 2000; Charbonneau et al. 2000) it has become clear that this is a viable search technique, applicable even to Earth-size planets in future space missions. Numerous programs using both small wide-field telescopes for bright stars and large-aperture telescopes for fainter stars have been established to monitor increasing numbers of objects with high photometric precision $(<1 \%)$ in a variety of stellar populations (see, e.g., Horne 2003, and references therein). The Optical Gravitational Lensing Experiment in its most recent incarnation (OGLE-III) is among the most successful of these searches, having uncovered 59 candidate transiting planets in its first observing season (2001) in three fields toward the Galactic center (Udalski et al. 2002a,b), another 62 in three other fields in the constellation of Carina during 2002 (Udalski et al. 2002c), and 16 additional candidates in the bulge and in Carina from a recent reanalysis of the original photometric observations (Udalski et al. 2003). All of these candidates show dips in the brightness of the star of only a few percent, 
and have photometric periods of a few days. Follow-up of the bulge sample has recently resulted in the spectroscopic confirmation of a second extrasolar transiting planet, around the star OGLE-TR-56, the first case originally discovered from its photometric signature rather than its Doppler signature (Konacki et al. 2003).

The success with HD $209458(V=7.65)$ has led to general optimism about the possibility of detecting significant numbers of transiting planets among similarly bright stars, and performing additional observations of the kind carried out for HD 209458 (e.g., Brown et al. 2001), which among other things led to the remarkable detections of the atmosphere of the planet (Charbonneau et al. 2002; Vidal-Madjar et al. 2003). More skeptical views have been voiced about the feasibility of high-precision Doppler follow-up of faint targets such as those uncovered by the OGLE project and others $(V=15-19)$. It has also been recognized that a variety of other phenomena can mimic transit light curves by producing shallow eclipses, and that these situations need to be examined carefully in order to be ruled out before a planet discovery can be claimed. Examples of these "false positives" include a stellar (as opposed to a substellar) companion orbiting a large star (B-A main sequence star, or a giant), grazing eclipses in a stellar binary, and contamination by the light of a fainter eclipsing binary along the same line of sight (referred to as a "blend").

So far no other transiting planets have been discovered among bright stars. OGLE-TR56 is a much fainter object $(V=16.6)$. Furthermore, from the results by Konacki et al. (2003) there are indications that the incidence of blends in searches among fainter stars in generally crowded fields is rather high (possibly as high as $98 \%$ ). Thus, discovering additional transiting extrasolar planets has turned out to be somewhat more difficult than anticipated (see also Charbonneau 2003).

In this paper we report our findings for several more targets from the OGLE-III sample towards the bulge, based on precise measurements of the radial velocities of these stars. We present the methodology and tests that document the stability of our instrumental setup, a key issue for confirming the reality of any Doppler signal. Results are given for OGLE-TR-10 and OGLE-TR-58, which remain good candidates for having a planetary companion in orbit around them, and additional supporting details for OGLE-TR-56 are given to supplement those reported by Konacki et al. (2003). We discuss also the case of OGLE-TR-3, recently claimed by Dreizler et al. (2003) to harbor a transiting planet, but we conclude that the evidence is actually against that interpretation, and more in favor of a blend. Another case of a blend, OGLE-TR-33, will be presented in full detail elsewhere. 


\section{Observations}

Because the OGLE targets are relatively faint, high-resolution follow-up of large numbers of them to detect their Doppler signatures becomes expensive or impractical in terms of telescope time. Thus, our strategy was to make use of all the information available from the photometry to eliminate obvious binary systems, and then to perform preliminary lowresolution spectroscopic observations ("reconnaissance") in order to rule out others, so that we may focus our attention for the high-precision velocity measurements only on the best candidates.

\subsection{Inspection of the light curves and low-resolution spectroscopic reconnaissance}

Light curves based on the photometry from the OGLE-III survey for all 59 targets in the direction of the Galactic bulge were examined carefully for any indications that might rule out the object as a good candidate. For example, we looked for signs of a secondary eclipse and/or out-of-eclipse variations that are strongly indicative of a massive (stellar) companion as opposed to a substellar companion.

Our initial inspection of the photometry allowed us to rule out a total of 20 candidates for a variety of reasons. Eight of them showed fairly obvious signs of stellar secondaries in the light curves. Four have only one transit detected, and thus the period is unknown. Another star is a duplicate entry (OGLE-TR-29 = OGLE-TR-8). A further 7 were considered too faint for follow-up and were also excluded, thus leaving us with 39 candidates from the original list. It should be noted here that since the time of our original inspection of the light curves, more sophisticated numerical methods have been developed to quantify their shape outside of eclipse (Drake 2003; Sirko \& Paczyński 2003). These lead to the rejection of a few more of the bulge candidates, although these same objects were also rejected on the basis of the low-resolution spectroscopy we describe in the following.

The 39 best candidates were observed spectroscopically at relatively low resolution with two different instruments. For the brighter objects we used the 1.5-m Tillinghast reflector at the F. L. Whipple Observatory on Mt. Hopkins (Arizona) in May and June 2002, with

the FAST spectrograph (resolving power $R \simeq 4400$ ). For the remaining objects we used the 6.5-m Baade telescope (Magellan I) at the Las Campanas Observatory (Chile) on July 17-21 2002 with the Boller \& Chivens spectrograph $(R \simeq 2200)$. One of the goals was to identify the early-type stars among the candidates, which are too large for an orbiting Jovian-size planet to produce a detectable drop in brightness, implying that the companion must be stellar. A 
handful of these cases were found in similar spectroscopic work at somewhat lower resolution by Dreizler et al. (2002) in the same sample. A further goal was to reject stellar binaries with grazing eclipses by measuring the radial velocities of the target stars multiple times. Stellar binaries will show variations of tens of $\mathrm{km} \mathrm{s}^{-1}$ due to orbital motion (which is large compared to the $2-3 \mathrm{~km} \mathrm{~s}^{-1}$ precision we achieved in these observations), as opposed to changes of only a few hundred $\mathrm{m} \mathrm{s}^{-1}$ for true planets. As a result of this reconnaissance, we established that 8 of the candidates are of early spectral type $(\mathrm{B}-\mathrm{A})$, and 25 showed velocity variations or even double lines in the spectra indicating that they are stellar binaries. Only 6 showed no significant variations within the errors, and were assigned the highest priority for the high-resolution follow-up described below. Further details of the low-resolution observations and complete information about all the rejected OGLE candidates will be reported elsewhere (Torres et al., in preparation).

\subsection{High-resolution spectroscopy and radial velocity measurements}

Five of our 6 best candidates were observed spectroscopically on 4 nights in July 24272002 with the Keck I telescope and the HIRES instrument (Vogt et al. 1994). These stars are OGLE-TR-3, OGLE-TR-10, OGLE-TR-33, OGLE-TR-56 and OGLE-TR-58. The setup allowed us to record 35 usable echelle orders covering the spectral range from $3850 \AA$ to $6200 \AA$ at a resolving power of $R \simeq 65,000$. Typical signal-to-noise ratios are in the range of 10-20 per pixel for a single exposure. Our main wavelength reference was provided by a hollow-cathode Thorium-Argon lamp, of which we obtained short exposures immediately preceding and following each stellar exposure. For two of the brighter objects (OGLETR-10 and OGLE-TR-58) we also made use of the iodine $\left(I_{2}\right)$ gas absorption cell - the standard setup for highly-precise Doppler planet searches with this instrument (e.g., Vogt et al. 2000) - which imprints a dense spectrum of $I_{2}$ lines directly on the stellar spectrum in the region from approximately $5000 \AA$ to $6200 \AA$ (some 12 echelle orders), and which serves as a very accurate and stable reference frame for measuring velocity shifts. Template exposures of these two stars without the iodine were also taken on one of the nights for use in the reduction process, although in the final iodine reductions we replaced them with synthetic templates $(\S 4)$.

In addition to our program stars we obtained frequent observations of two brighter stars (HD 209458 and HD 179949) that have known low-amplitude velocity variations at the level of about $200 \mathrm{~m} \mathrm{~s}^{-1}$ due to orbiting substellar companions, and which we used as "standards". These stars were observed with the iodine cell. Additionally we observed a number of 10th-magnitude K stars repeatedly for a different observing program, also with the 
iodine cell, which turned out to be extremely useful as secondary standards for monitoring the stability of the velocity zero point and verifying systematics for fainter stars. Identifiers and observational details including exposure times for all objects discussed here are given in Table 1. All HIRES spectra were bias-subtracted, flat-fielded, cleaned of cosmic rays, and extracted using the MAKEE reduction package written by Tim Barlow (2002). Wavelength solutions based on the Th-Ar exposures were carried out with standard tasks in IRAF ${ }^{5}$.

Radial velocities for OGLE-TR-3, OGLE-TR-33, and OGLE-TR-56 relied on the Th-Ar calibration exposures for the wavelength reference. These are referred to as 'Th-Ar velocities'. For the other two targets we used the iodine as the reference ('iodine velocities'). ThAr velocities were derived by cross-correlation against synthetic templates described below, using the IRAF task RVSAO (Kurtz \& Mink 1998). The final velocities are the weighted average of all echelle orders. Formal errors were derived from the scatter of the velocities determined from the different orders. These are typically under $\sim 100 \mathrm{~m} \mathrm{~s}^{-1}$, and do not include systematic components discussed later, which we also estimate to be no larger than about $100 \mathrm{~m} \mathrm{~s}^{-1}$. The velocities in the frame of the solar system barycenter and their internal errors are listed in Table 2.

For OGLE-TR-10 and OGLE-TR-58 we used iodine reduction software (see, e.g., Korzennik et al. 2000) with which we have typically achieved precisions of $\sim 10 \mathrm{~m} \mathrm{~s}^{-1}$ or better for a single measurement on bright stars. For our much fainter OGLE targets the limit to the final precision in the iodine velocities is signal to noise, not systematics. These iodine velocities are reported in Table 3, and include barycentric corrections. For these targets and also for the standard stars the comparison between the Th-Ar velocities and the iodine velocities allows us to estimate the accuracy of the Th-Ar technique, as applied to the OGLE candidates that were observed without iodine.

\subsubsection{Stellar parameters and synthetic spectra}

The key physical properties of each of our program stars were estimated from our highresolution Keck spectra, by comparison with calculated spectra. These were computed from model atmospheres for solar composition based on the ATLAS 9 and ATLAS 12 programs by Kurucz (1995), re-written in Fortran-90 (J. Lester, priv. comm.) and incorporating new routines for improved treatment of contributions from various broadening mechanisms, as well as updated and expanded opacities and line lists. This code has been tested extensively

\footnotetext{
${ }^{5}$ IRAF is distributed by the National Optical Astronomy Observatories, which is operated by the Association of Universities for Research in Astronomy, Inc., under contract with the National Science Foundation.
} 
and performs very well for solar-type stars (F-K-type) such as our targets. All spectra for each OGLE target (both the template spectra with no iodine, and the iodine exposures) were shifted to a common reference frame and co-added. A comparison between the observed coadded spectra and the synthetic spectra computed for a range of stellar parameters was made in spectral regions unaffected by the $I_{2}$ lines, and including a large number of metal absorption lines of different ionization and excitation states as well as the core and wings of the $\lambda 4861 \mathrm{H} \beta$ line. Effective temperatures $\left(T_{\text {eff }}\right)$ were determined to an estimated accuracy of about $100 \mathrm{~K}$, surface gravities $(\log g)$ to roughly \pm 1 dex, metallicity $[\mathrm{Fe} / \mathrm{H}]$ to \pm 0.3 dex, and projected rotational velocities $(v \sin i)$ to $2-3 \mathrm{~km} \mathrm{~s}^{-1}$. Synthetic templates with these parameters (and degraded to the resolution of our Keck observations) were used for all crosscorrelations in order to obtain radial velocities. In Figure 1 we show a section of the co-added spectra for each of our stars in the $\mathrm{H} \beta$ region, as well as the best-fit synthetic spectrum and the values we derive for the stellar parameters. In addition to comparing and fitting metal lines of different ionization states and excitation potentials (mainly for $T_{\text {eff }}$ and $[\mathrm{Fe} / \mathrm{H}]$ ), we carefully fit the core vs. wings of the Balmer $\mathrm{H}$ lines. Our approach is similar to that described by Fischer \& Valenti (2003) regarding line broadening.

The metal abundance for all our targets is consistent with the solar value. Other derived properties such as masses and radii for our stars, used later in the analysis, were estimated with a stellar evolution code described in detail elsewhere (Cody \& Sasselov 2002; Sasselov 2003). Since the distance to our targets is not known accurately, we used $\log g$ as a proxy for luminosity. Evolutionary tracks are nearly vertical in the $T_{\text {eff }}$ vs. $\log g$ plane for our relatively low-mass stars, so the fairly large uncertainties in the surface gravity do not affect the inferred mass significantly for our purposes.

\section{Random and systematic errors in the Th-Ar radial velocities: velocity standards and HIRES stability}

One of the key requirements for the measurement of highly precise radial velocities is a reliable reference frame that enables one to map small pixel shifts in the stellar lines into wavelength shifts. To achieve $\mathrm{ms}^{-1}$ precision with HIRES spectra, the shifts that need to be measured are of the order of a few thousandths of a pixel. In the classical approach that uses a comparison lamp, the accurate measurement of velocities requires that the calibration spectrum present a large number of suitable lines with which to compute a wavelength solution, but most importantly, that there be no systematic shifts between the stellar exposure and the lamp exposure(s). The latter is the most serious limitation for HIRES, as we show in this section, since the comparison spectra can only be taken before 
or after the stellar spectrum. If shifts do occur, they can in principle still be corrected for as long as they can be modeled accurately (e.g., shifts that are linear with time). The power of the iodine technique comes from the fact that a very dense forest of $I_{2}$ lines is imprinted simultaneously with the stellar spectrum, thus allowing shifts (and higher-order distortions) of the wavelength scale to be measured accurately. However, the use of the iodine cell decreases the signal from the stellar spectra by a large factor (see $\S 4$ ), which is a serious drawback for faint objects. Thus for most of the stars in our sample we were forced to rely on the Th-Ar exposures for the wavelength reference.

To minimize shifts such as those mentioned above, each exposure of a program star was bracketed by Th-Ar exposures taken immediately before and after the stellar exposure. In addition, velocity standards were observed frequently to allow us to monitor residual instrumental shifts. Because these standard stars are much brighter than our targets, they were observed with the iodine cell to take full advantage of that technique. With our setup, approximately one third of the echelle orders have $I_{2}$ lines and two thirds do not, so that the orders unaffected by iodine can be used in the classical way for the standard stars to obtain radial velocities using the Th-Ar lamp as the reference.

The standard stars were used for two main purposes: (1) To assess the magnitude of the shifts that can occur between the Th-Ar spectra and the stellar spectra on timescales similar to the exposures for the OGLE targets (typically $\sim 30$ minutes); and (2) to estimate the intrinsic accuracy of the wavelength solutions based on the lamp exposures and its impact on the velocities.

An indication of instrumental shifts that can occur on timescales of hours can be obtained by comparing the pixel locations of the emission lines in the Th-Ar spectra taken during the night. For this, we cross-correlated each lamp spectrum in pixel space against one of them adopted as the reference, using the IRAF task FXCOR, and computed the weighted average over all orders. These shifts are shown with filled symbols in Figure 2, as a function of time. Typical errors for an individual shift are 0.005-0.009 pixel. Additional information on shifts throughout the night can be obtained from the iodine spectrum imposed on the bright standard stars and on the $\mathrm{K}$ stars. The high density of $I_{2}$ lines allows these shifts to be determined very accurately relative to one of such spectra adopted as the reference. Aside from an arbitrary offset with respect to the shifts measured from the Th-Ar exposures that is easily removed, the iodine shifts are seen to follow a similar trend as the Th-Ar shifts within each night (Figure 2, open symbols). It is clear from the figure that there are significant shifts with time within any given night and often with each new telescope pointing, although the magnitude of these shifts is not entirely predictable. We point out also that the shifts from night to night are not always meaningful, as the position 
of the detector was occasionally adjusted manually (only at the beginning of the night) to keep the Th-Ar lines within $\sim 0.5$ pixel of a nominal location on the CCD. Shifts are seen to be generally less than 0.5 pixel during a night, but even this corresponds to a very significant shift in velocity space $\left(1 \mathrm{~km} \mathrm{~s}^{-1}\right)$, making it imperative to obtain Th-Ar exposure before as well as after each stellar exposure.

A key assumption in the Th-Ar analysis is that the shift between the two comparison exposures is linear with time, so that interpolation to the mid-time of the stellar exposure is essentially free from systematics. Demonstrating that this is actually the case by taking long sequences of exposures under actual observing conditions would be excessively timeconsuming. However, given that these shifts seem unavoidable, we can at least place useful limits on their residual systematic effect by considering the largest shift that occurred during our run. In Figure 3 we show the sequence of Th-Ar exposures on each night, enlarged from Figure 2, and we indicate with vertical dotted lines the times of our interleaved exposures of the OGLE candidates. Consecutive exposures of the same candidate are usually intended for cosmic ray removal, or correspond to iodine-template pairs. The largest shift between Th-Ar exposures occured on the last of our nights (for OGLE-TR-58), and corresponds to 0.4 pixel over a 2-hour interval. This represents a velocity shift of about $200 \mathrm{~m} \mathrm{~s}^{-1}$ over 30 minutes, which is the typical exposure time for our targets. Since the interpolated wavelength solutions we adopt are for the nominal center of each exposure, we may assume that systematic errors will typically be half of the total shift, or $\sim 100 \mathrm{~m} \mathrm{~s}^{-1}$ (0.05 pixel) in this case. We adopt this as a conservative estimate of our systematics for this run, although shifts for other stars in our sample are probably smaller.

The intrinsic accuracy of the Th-Ar technique was explored by observing two bright standard stars (HD 209458 and HD 179949) multiple times during the run, often 2 or 3 times during the same night. In addition we observed more than a dozen 10th-magnitude $\mathrm{K}$ stars for a separate project. All of these objects were observed with the iodine cell, and also once without the iodine cell to provide a template. Initially we did not anticipate reducing them in the classical manner, so no Th-Ar exposures were obtained either before or after these stellar exposures. Nevertheless, they provide useful checks as we describe below.

Six of the $\mathrm{K}$ stars showed no iodine velocity variations at the level of $10 \mathrm{~m} \mathrm{~s}^{-1}$, and are therefore suitable as standards for our purposes (see Table 1). Th-Ar velocities for these stars were obtained from the non-iodine orders, by cross-correlation against the corresponding noniodine exposures used as templates. The wavelength reference adopted was that provided by the first Th-Ar exposure of the night (same for all stars on each night). The Th-Ar velocities for each of the $\mathrm{K}$ stars during a given night contain an unknown systematic error that has at least two components: an unknown instrumental shift occuring between the time of the 
stellar (iodine) exposure and the Th-Ar exposure at the beginning of the night, and another similar shift associated with the wavelength calibration of the observed template (which was not necessarily obtained on the same night). The latter component is of course irrelevant when comparing velocities of the same star, since the template is always the same. We may thus represent the measured Th-Ar velocity as

$$
V_{i}\left(t_{j}\right)=\hat{V}_{i}\left(t_{j}\right)+W_{i}\left(t_{j}\right), \quad i=1, \ldots, 6, \quad j=1, \ldots, 4
$$

where $V_{i}\left(t_{j}\right)$ is the velocity affected by systematics and $\hat{V}_{i}\left(t_{j}\right)$ is the true velocity of the $i$-th star taken on the $j$-th night. If we now use one of the stars (e.g., star number 1) as the reference, we have

$$
\hat{V}_{i}\left(t_{j}\right)-\hat{V}_{1}\left(t_{j}\right)=V_{i}\left(t_{j}\right)-V_{1}\left(t_{j}\right)-\left[W_{i}\left(t_{j}\right)-W_{1}\left(t_{j}\right)\right], \quad i=2, \ldots, 6, \quad j=1, \ldots, 4 .
$$

Because the spectra of these $\mathrm{K}$ stars also have iodine lines in the orders redward of about $5000 \AA$, the differential shift $W_{i 1}\left(t_{j}\right) \equiv W_{i}\left(t_{j}\right)-W_{1}\left(t_{j}\right)$ can be determined very precisely (to $\sim 10 \mathrm{~m} \mathrm{~s}^{-1}$ ) using the iodine reduction software, and the systematic errors mentioned above can effectively be removed resulting in the Th-Ar velocity difference $\hat{V}_{i}\left(t_{j}\right)-\hat{V}_{1}\left(t_{j}\right)$. This allows us to evaluate the intrinsic precision of the Th-Ar technique, essentially free from systematics due to instrumental shifts. For this test we adopted HIP 1334 as star number 1 , and subtracted its velocity on each night from those of the remaining five $K$ dwarfs. In Figure 4 we display these differences $\hat{V}_{i}\left(t_{j}\right)-\hat{V}_{1}\left(t_{j}\right)$ after removing the average for each star (to account for the difference in absolute velocities between star $i$ and star 1). The scatter of these differences, $49 \mathrm{~m} \mathrm{~s}^{-1}$, is a measure of the uncertainties intrinsic to the wavelength calibrations in the classical Th-Ar technique, and the corresponding share for a single measurement is about $35 \mathrm{~m} \mathrm{~s}^{-1}\left(49 \mathrm{~m} \mathrm{~s}^{-1} / \sqrt{2}\right)$. This estimate reflects uncertainties that have to do with rebinning in the cross-correlation process, template mismatch, and other numerical uncertainties, and represents the absolute limit of this technique for the HIRES instrument in the absence of other shifts between the Th-Ar exposures and the stellar exposure, discussed above.

As a further test of the intrinsic uncertainties of the Th-Ar technique, as well as of our ability to measure small changes in velocity over our 4-night run, we used our spectroscopic observations of HD 209458 and HD 179949, which are known to have low-mass orbiting companions. Because the spectroscopic orbits of these stars are known (Mazeh et al. 2000; Tinney et al. 2001), they allow us to study any biases that may affect the determination of the velocity amplitudes. In addition, their planetary companions are in the mass range that we expect to be able to detect around the OGLE candidates, making them excellent test cases. As in the analysis with the K stars, we measured the radial velocities of HD 209458 and HD 179949 from the non-iodine orders using the first Th-Ar exposure of the night as the 
wavelength reference and the exposure without iodine as the template, and then subtracted the Th-Ar velocities of HIP 1334 and applied the corrections $W_{i 1}\left(t_{j}\right)$. These differences are shown in Figure 5 (filled circles). The intrinsic precision of a single Th-Ar measurement, as estimated from the scatter for HD 209458 and HD 179949, is $35 \mathrm{~m} \mathrm{~s}^{-1}$ and $29 \mathrm{~m} \mathrm{~s}^{-1}$, respectively (from $49 \mathrm{~m} \mathrm{~s}^{-1} / \sqrt{2}$ and $41 \mathrm{~m} \mathrm{~s}^{-1} / \sqrt{2}$ ), which are similar to our result for the $\mathrm{K}$ stars. It is worth noting that this similarity is an indication that photon statistics is not the dominant factor in the estimate, since there is roughly a factor of two difference in $\mathrm{S} / \mathrm{N}$ between these two bright stars and the $\mathrm{K}$ dwarfs: the latter are $\sim 3$ mag fainter, and have exposures that are 5 times longer.

A similar exercise with the two brighter standards but subtracting one from the other is shown in Figure 6, where the differential orbit is computed from the orbital elements of the two stars. This is analogous to Figure 2 by Konacki et al. (2003), except that the corrections $W_{i 1}\left(t_{j}\right)$ (where star 1 is HD 179949) have now been applied to the differential ThAr velocities. The residual scatter from the computed velocity difference (solid curve) is 69 $\mathrm{m} \mathrm{s}^{-1}$ (corresponding to $49 \mathrm{~m} \mathrm{~s}^{-1}=69 \mathrm{~m} \mathrm{~s}^{-1} / \sqrt{2}$ for a single velocity). For comparison, the scatter without the corrections is $97 \mathrm{~m} \mathrm{~s}^{-1}$, giving an error of $69 \mathrm{~m} \mathrm{~s}^{-1}$ for a single velocity.

From the above discussion we conclude that the intrinsic errors of the Th-Ar technique with HIRES (unaffected by systematics) are smaller than about $50 \mathrm{~m} \mathrm{~s}^{-1}$ per measurement. An additional component to the error for the OGLE stars comes from the lower signal-tonoise ratio of their spectra compared to those of bright stars. We estimate this contribution to be around $60 \mathrm{~ms}^{-1}$ (see next Section). Finally, the main contributor to the overall error comes from the systematic shifts (and their non-linearity) that we cannot control, between the lamp exposures and the stellar exposures, which appear to occur on timescales similar to the duration of the target exposures. We have conservatively estimated this to be $\sim 100$ $\mathrm{ms}^{-1}$, although in most cases it should be less (see above).

Thus our experiments show that, with appropriate observing protocols, radial-velocity follow-up observations of objects as faint as $V=16-17$, such as those from the OGLE-III survey, can achieve the required precision of $\sim 100 \mathrm{~m} \mathrm{~s}^{-1}$ to detect close-in extrasolar giant planets using the classical Th-Ar technique.

\section{Radial velocities with the iodine cell}

As seen in the previous section, the fundamental limitation of HIRES for obtaining very accurate radial velocities with the classical Th-Ar technique is the unavoidable drifts in the location of the spectrum on the detector that occur throughout the night, on timescales of 
hours or less. In particular, non-linearity of these shifts between the time of the preceding and following comparison lamp exposures is essentially impossible to account for. However, other instrumental setups that rely on comparison lamps can overcome this limitation, such as fiber-fed spectrographs that are designed to record Th-Ar spectra interleaved with the stellar spectra during the same exposure. As an example, the CORALIE spectrograph on the 1.2-m Swiss telescope at the European Southern Observatory can regularly achieve errors of 10-15 $\mathrm{m} \mathrm{s}^{-1}$ or better with this technique (see, e.g., Udry et al. 2000). In the case of HIRES on the Keck I telescope, a popular alternative is the use of the iodine gas absorption cell, as described earlier, which can track shifts very accurately. This method is capable of reaching very high precision (and accuracy) of a few $\mathrm{ms}^{-1}$ (e.g., Butler et al. 1996) by carefully modeling the instrumental profile of the spectrograph (often referred to as the point-spread function, or PSF) explicitly, but it requires very high $\mathrm{S} / \mathrm{N}$ ratios (typically 100 or more) in order to achieve this. Unfortunately, the absorption from the iodine cell effectively removes up to one half or even two thirds of the light from the star, depending on the spectral type, and it is generally considered that the approach becomes impractical for stars fainter than about $V=13$. It is particularly onerous not only because of the long exposures needed for faint stars with the iodine cell, but also because of the even longer exposures required for the templates, which must ideally be of much better quality so that their noise characteristics do not completely undermine the entire procedure.

However, if main goal is to limit the systematic errors (instrumental shifts) that are at the level of $\sim 100 \mathrm{~m} \mathrm{~s}^{-1}$ or so for HIRES, the iodine-cell technique can still be used to advantage on faint stars such as our OGLE candidates with a few modifications, as follows:

(1) The most important factor that limits the velocity errors at the level of $10 \mathrm{~m} \mathrm{~s}^{-1}$ for bright stars is the variability of the PSF with time and even with position on the CCD. Thus modeling the spectrograph PSF very carefully is a central feature of all iodine reduction packages. Given that the orbital periods of our OGLE candidates are typically short (a few days), the expected velocity variations due to planets similar to Jupiter are usually several hundred $\mathrm{m} \mathrm{s}^{-1}$, rather than tens of $\mathrm{m} \mathrm{s}^{-1}$, and therefore we do not require such high precision (which would be difficult to obtain for faint stars, in any case, because of S/N limitations). This allows us to simplify the iodine reduction software considerably by not modeling the PSF at all, and only adopting an estimate for it.

(2) The use of observed templates for each star in the standard iodine reductions is another important feature that allows the method to reach very high precision in the velocities, because it removes any spectral mismatch in modeling the iodine spectra. Since obtaining a template exposure for each OGLE target with sufficient $\mathrm{S} / \mathrm{N}$ would be very expensive in terms of telescope time, we can avoid this altogether by using calculated spectra instead. 
Two of our planet transit candidates were observed at Keck with the iodine cell, anticipating that the analysis with that technique might yield useful results: OGLE-TR-10 and OGLE-TR-58. In order to evaluate the impact of the simplifications in the iodine reduction described above, we have again made use of our spectra of HD 209458 and HD 179949. Synthetic templates for these stars were computed as described in $\S 2.2 .1$, with physical parameters as reported in the literature. The resulting velocities for the two stars are shown in Figure 7 (solid symbols) and are compared with the known orbits (Mazeh et al. 2000; Tinney et al. 2001). The standard deviation of the residuals is $26 \mathrm{~m} \mathrm{~s}^{-1}$ for HD 209458 and $22 \mathrm{~m} \mathrm{~s}^{-1}$ for HD 179949. These values are similar to the internal (formal) errors reported by our software, suggesting that the procedure is effective in removing systematic errors at this level. The contribution from photon noise is expected to be minimal $\left(<5-10 \mathrm{~m} \mathrm{~s}^{-1}\right)$ given the large $\mathrm{S} / \mathrm{N}$ ratios of the spectra for these stars in the iodine orders, which are in the range 100-300 per pixel. Thus, most of the error presumably comes from the simplifications introduced in the analysis (no PSF modeling, and template mismatch). As an illustration, the velocities computed using the observed templates instead of synthetic templates are represented with open circles in Figure 7. Their scatter is $13 \mathrm{~m} \mathrm{~s}^{-1}$ for HD 209458 and $10 \mathrm{~m} \mathrm{~s}^{-1}$ for HD 179949, showing the level at which template mismatch makes a difference.

When the same procedure (using synthetic templates) is applied to OGLE-TR-10 and OGLE-TR-58, which have much weaker spectra ( $\mathrm{S} / \mathrm{N}$ in the range $15-25$ in the iodine orders), the internal errors are typically $50-60 \mathrm{~m} \mathrm{~s}^{-1}$ (see Table 3). Based on the results for the brighter stars HD 209458 and HD 179949, and under the assumption that the combined effects of systematics and other simplifications in the method are similar for the OGLE stars and the brighter stars (i.e., at the level of $20-30 \mathrm{~m} \mathrm{~s}^{-1}$ ), we conclude that photon noise is the dominant factor in the iodine velocity errors for the OGLE targets. Thus the errors listed in Table 3 provide realistic estimates of the total uncertainties. These results are very encouraging, and suggest the method may be very useful in the future for the spectroscopic follow-up of other faint transiting planet candidates, from the OGLE survey and other similar transit searches.

\section{Analysis and discussion}

High-precision radial velocity measurements such as those reported above are crucial for establishing whether a star with a transit-like light curve is orbited by a planetary companion, but they are generally not sufficient because of the variety of and frequency with which false positives occur (e.g., Queloz et al. 2001; Yee et al. 2002; Latham 2003). The lack of detection

of any significant velocity change at the level of $100 \mathrm{~m} \mathrm{~s}^{-1}$ does not necessarily prove there 
is a substellar companion in orbit, since the star may actually have a constant velocity, but be blended with a much fainter eclipsing binary along the same line of sight that is invisible in the spectrum and is the object responsible for the shallow dips in brightness seen photometrically. The opposite case, the detection of a small velocity variation, still does not constitute absolute proof of a planetary companion and must be investigated carefully to rule out other possible reasons for the apparent change. For example, if the primary of a contaminating background eclipsing binary is bright enough, its spectral lines would move back and forth with the photometric period and could produce slight asymmetries in the spectral lines of the main star that vary with phase. These, in turn, might lead to completely spurious small-amplitude velocity variations. Therefore, in addition to measuring the Doppler shifts for each of our OGLE targets, we have examined the profiles of the spectral lines looking for strong asymmetries or asymmetries that vary in phase with the photometric period.

The cross-correlation analysis of the Keck spectra produces a correlation function for each echelle order, which we used to derive the Th-Ar velocities. The sum of these correlation functions is representative of the shape of average line profile of the star. Asymmetries for each spectrum were measured by computing the line bisectors (e.g., Gray 1992), and are shown in Figure 8 for OGLE-TR-3, OGLE-TR-10, OGLE-TR-56, and OGLE-TR-58. Line asymmetries are present in all of these stars, but they are not significantly larger than in the Sun (in which the velocity span is typically a few hundred $\mathrm{m} \mathrm{s}^{-1}$ ). Given that the stars themselves are quite similar to the Sun in effective temperature, we conclude that these asymmetries are not unusual. Furthermore, they do not show any obvious changes with photometric phase, which suggests the Doppler shifts are not significantly affected.

It is well known that chromospheric activity can induce spurious changes in the velocity of a star, as well as in the photometry, that are unrelated to the presence of an orbiting companion. We have examined the profiles of the Ca II H and K lines for each of our targets for any signs of emission that might indicate high levels of activity. Although the strength of the spectra at these wavelengths is typically low, we see no obvious indications of emission in any of the OGLE stars.

Information from the light curves is also very helpful in ruling out false positives. For example, the shape and duration of the transits (ingress-egress, and flat portion) provides some constraint on whether the companion can be a planet (see Seager \& Mallén-Ornelas 2003), although it is highly dependent on the quality of the light curve. The overall shape of the light curve outside of eclipse also provides important clues (see below). We have applied the prescriptions by Seager \& Mallén-Ornelas (2003) to our OGLE targets to establish whether the nature of the primary stars as inferred directly from the transit light curves is 
consistent with the properties we derive from our spectra (mainly the effective temperature and surface gravity). Although formally most stars pass the test, the uncertainties are such that in general the results are inconclusive.

In the following we examine the spectroscopic and photometric evidence available for each of our candidates.

\subsection{OGLE-TR-3: a probable blend}

Very recently a claim has appeared in the literature that OGLE-TR-3 harbors a possible new transiting planet (Dreizler et al. 2003). These authors obtained 10 radial velocity measurements over a period of 26 days in 2002, coincidentally the same month as our own observations, using the UVES instrument on the 8.2-m VLT with a typical precision of $\sim 100$ $\mathrm{m} \mathrm{s}^{-1}$ per measurement. They examined three alternative scenarios not involving a planet that might explain the available observations. Two of these scenarios invoke blends with an eclipsing binary along the same line of sight with $P=1.19 \mathrm{~d}$ (the photometric period determined by Udalski et al. 2002a), and another proposes that the true photometric period is actually twice the value reported by Udalski et al. (2002a), and involves also a blend with an eclipsing binary composed of two equal-size M stars. Dreizler et al. (2003) concluded that none of these scenarios is as satisfactory as the planet hypothesis.

Unfortunately our own velocity measurements of OGLE-TR-3 with the Keck telescope do not allow us to test this claim, since we were only able to observe the star on two nights, and the precision of the velocities on one of them is rather low (see Table 2). Within the errors we detect no significant change. Nevertheless, we can place other useful limits by examining line asymmetries and also looking for the presence of a second set of lines in our spectra, which might typically be expected in the case of a blend. As indicated earlier, the spectral line bisectors shown in Figure 8a do not indicate any significant asymmetries (the span of the bisectors is less than $\sim 200 \mathrm{~ms}^{-1}$ ), and they show no correlation with the phase in the orbit (i.e., the photometric phase). Similar conclusions on the lack of asymmetries were reached by Dreizler et al. (2003) using their own data. Inspection of our spectra using the two-dimensional correlation technique TODCOR (Zucker \& Mazeh 1994) to search for signs of a second set of lines (presumably the main star of the blended eclipsing binary) did not reveal anything significant at the level of $5 \%$ of the light of the primary within \pm 200 $\mathrm{km} \mathrm{s}^{-1}$ of its mean velocity. This represents a significantly stronger constraint than the $30 \%$ limit reported by Dreizler et al. (2003).

Although the previous paragraph appears to support the Dreizler et al. (2003) claim in 
finding no evidence for a blend, careful examination of their methods and observations lead us to conclude that some of the evidence they presented in favor of a planet is not quite correct, and a more complete analysis of all the data argues against the reality of a planet.

For the analysis of their radial velocity measurements Dreizler et al. (2003) adopted the photometric ephemeris for transits published by Udalski et al. (2002a), which is $T=$ $2,452,060.22765+1.18990 \cdot E$, where $E$ is the number of cycles from the reference epoch of transit. An updated ephemeris based on a total of 14 recorded transits is available from the OGLE website ${ }^{6}$,

$$
T=2,452,060.24529+1.18917 \cdot E,
$$

and is significantly better as can be seen easily from the tightness of the folded photometric data, although it does not significantly affect their conclusions — or ours below ${ }^{7}$.

Dreizler et al. (2003) state that they solved for the spectroscopic orbital elements by holding the period fixed, and adjusting the velocity offset ( $\gamma$ in our notation, see eq. 4 ), the velocity semi-amplitude $K$, and also a phase shift, $\Delta \phi$. Given that the precision of their velocity measurements is of the same order as the amplitude they attempted to fit, and that the number of measurements is not large, allowing for a phase shift instead of relying on the much better determined photometric epoch of transit is highly inadvisable. The available radial velocities (a few of which have errors as large as $0.6 \mathrm{~km} \mathrm{~s}^{-1}$ ) provide essentially no useful constraint on the phase of OGLE-TR-3. In describing their velocity fit (illustrated in their Figure 7), the authors make the statement that "As expected, the derived radial velocity is indeed zero at mid eclipse". While this is true, the slope of their radial velocity curve at phase 0.0 is positive instead of negative, clearly showing that this velocity curve is in fact inconsistent with the transits. By adopting the following model

$$
V_{\star}=\gamma-K \sin (2 \pi[\phi(t)+\Delta \phi])
$$

where $\phi(t) \in[0,1]$ is the orbital phase determined from photometry (through equation 3), we are able to reproduce their results for the amplitude and velocity offset using their measured Doppler shifts. However, the phase shift we obtain is $\Delta \phi=0.55 \pm 0.05$ rather than the value of 0.02 listed in their Table 2 from their $\chi^{2}$ fit, possibly due to the use by Dreizler et al. (2003) of a positive sign in front of $K$ (which is in fact inconsistent with the photometric ephemeris). Nevertheless, a plot of the velocities and computed curve from our solution looks

\footnotetext{
${ }^{6}$ http://bulge.princeton.edu/ ogle/ogle3/transits/tab.html

${ }^{7}$ The original ephemeris is based on the photometry from the first of the OGLE observing seasons, JuneOctober 2001. The OGLE data from the following year folded with the same period show an offset of about 0.2 in phase, which disappears when using the new period.
} 
essentially the same as the one in their Figure 7, confirming that for such fit there is indeed a shift of about half a cycle compared to the photometric ephemeris, and this explains the reversal of the slope at the primary eclipse.

We believe the only sensible approach in a case such as this is to adopt the phasing from the photometry (the updated ephemeris in eq. 3), and to solve only for $\gamma$ and $K$. This solution results in a value of $K$ that is statistically insignificant: $K=0.100 \pm 0.061 \mathrm{~km} \mathrm{~s}^{-1}$. A graphical representation of the data and this fit is shown in Figure 9a.

This alone does not necessarily prove that there is not a planet in orbit around the central star. It only shows that the star has no significant velocity variation within the errors. However, strong hints (also admitted by Dreizler et al. 2003) of a secondary eclipse at phase 0.5 in the light curve of OGLE-TR-3 suggest otherwise ${ }^{8}$. This is seen in Figure 9b, which is based on the original OGLE photometry along with the ephemeris in eq. 3. More objective ways of assessing the reality of a secondary eclipse (or more generally, of the curvature outside of eclipse due to ellipsoidal variations - the telltale sign of a stellar companion) support these indications. For example, Drake (2003) carried out analytical fits of the form $\cos 2 \phi$ (sinusoidal modulation at twice the orbital frequency) and concluded that the amplitude of the out-of-eclipse variation in OGLE-TR-3 is significant (1.49 $\pm 0.37 \mathrm{mmag})$. More recently Sirko \& Paczyński (2003) improved on the analysis and accounted also for correlations in the OGLE photometry that they claimed led Drake to underestimate the errors. They too concluded that the amplitude of the ellipsoidal variation is significant (2.67 $\pm 0.67 \mathrm{mmag})$, and also found a significant heating (reflection) effect for OGLE-TR-3 of $2.58 \pm 0.73 \mathrm{mmag}$, another indication of the stellar nature of the companion. On this basis they ruled it out as a good candidate for planetary transits.

Given the limits described at the beginning of this Section on possible blend scenarios, we carried out extensive simulations in an attempt to find configurations that are consistent with all the observational evidence available for OGLE-TR-3. The techniques are introduced and described in detail by Torres et al. (2003, in preparation), and consist essentially of fitting the observed light curve with a full-fledged eclipsing binary light curve solution program constraining the stars to have physical properties derived from theoretical isochrones and stellar evolution models. The isochrones are the same for the eclipsing binary and the third star if they form a physical triple system, or otherwise they can be different. The properties of OGLE-TR-3 itself (effective temperature, rotational velocity, estimate of the

\footnotetext{
${ }^{8}$ This feature was overlooked in the initial assessment of the photometry that led to the placement of OGLE-TR-3 on our Keck observing program. In retrospect, the star should not have been considered as a good candidate.
} 
surface gravity) are constrained to agree with our values for these quantities reported earlier. Testable predictions can be made regarding the relative brightness of the stars, the velocity amplitudes $K_{1}$ and $K_{2}$ of the eclipsing binary, and the rotational velocities $v \sin i$ of its components (assuming rotation is synchronized with orbital motion, presumably valid for short periods). We confirmed the claim by Dreizler et al. (2003) that a blend with an eclipsing pair of equal $\mathrm{M}$ dwarfs having a period that is twice the reported period results in eclipses that are too narrow (see their Figure 11). We also explored configurations where a grazing eclipsing binary forms a physical triple system with the main star we see in OGLE-TR-3, but in this case the relative luminosity of the primary of the eclipsing pair is predicted to be $40-50 \%$ of the light of the third star in the optical, which we can clearly rule out from our spectra (see above). However, a situation in which the grazing eclipsing binary is in the background and has its eclipses diluted by OGLE-TR-3 can produce an acceptable fit (reduced $\chi^{2}=1.03$ ) and gives a predicted relative optical luminosity for the primary star in the binary of the order of $4 \%$ or less, which is below the detection threshold in our spectra. The difficulty in detecting the spectral signature of such a background star is compounded by the expected $v \sin i$ of $\sim 75 \mathrm{~km} \mathrm{~s}^{-1}$, which smears out the spectral lines considerably. The contribution of the foreground star in this simulation (OGLE-TR-3 itself) is $89 \%$ of the total light in the $I$ band, comparable to estimates illustrated by Dreizler et al. (2003) in their Figure 12. A sample fit to the light curve for this particular blend case is shown in Figure 10.

The a priori likelihood of such a blend scenario, in which the eclipsing binary is aligned with OGLE-TR-3 to within about $1^{\prime \prime}$ (the resolution limit of the photometry) may not be very high, but it cannot be ruled out completely, particularly in a very crowded field such as toward the Galactic center. Given the evidence presented above (i.e., signs of a secondary eclipse, and consistency with a blend model having a grazing eclipsing binary in the background) along with the lack of any significant velocity variations, we must conclude as stated by Konacki et al. (2003) that based on all the data available OGLE-TR-3 is most likely not orbited by a transiting planetary companion.

\subsection{OGLE-TR-10: planetary companion or blend}

Of the three spectra we obtained for OGLE-TR-10 using the iodine cell, one is too weak to be usable and the other two show no significant velocity variation within the errors, over an interval of two days (Table 3). The line asymmetries are not unusually large and show no

obvious trend with phase, as seen in Figure 8b. Careful examination of the spectra reveal no sign of another star brighter than about $5 \%$ of the light of the primary. Also, the analysis 
by Sirko \& Paczyński (2003) indicates that the out-of-eclipse variations in the light curve are insignificant.

In principle all of this evidence allows for the possibility of a substellar companion. In fact, the formal velocity difference between the two available measurements is consistent with the expected trend from the most recent ephemeris $(P=3.10140$ days; see footnote 6$)$, which is based on 7 or possibly 8 transits (see Figure 11). As an exercise, a determination of the velocity amplitude through

$$
K=\left[V_{\star}\left(\phi_{1}\right)-V_{\star}\left(\phi_{2}\right)\right] /\left(\sin \phi_{2}-\sin \phi_{1}\right)
$$

results in the value $K=100 \pm 43 \mathrm{~m} \mathrm{~s}^{-1}$ (where the error was computed by propagating from the measurement errors using the above equation), and a corresponding companion mass of $0.7 \pm 0.3 \mathrm{M}_{\mathrm{Jup}}$, well within the planetary regime. An analysis of the OGLE-III light curve leads to an estimate of the radius of the companion of $\sim 1.3 \mathrm{R}_{\text {Jup }}$.

The above is of course insufficient to demonstrate that the companion is a planet, and a blend configuration could still account for all the observations. In Figure 12 we show the results of our blend simulation for this system, illustrating the fit for one such scenario in which the star OGLE-TR-10 dilutes the light of a background eclipsing binary comprised of an M1 main sequence star in orbit around a G0 star, with the photometric period determined by the OGLE team. In this case the orbit of the binary is exactly edge-on, and the brightness of the G0 star in the optical is only $3 \%$ of that of OGLE-TR-10, which is below the detection threshold in our spectra. OGLE-TR-10 contributes $94 \%$ of the total light in the $I$ band. A very shallow secondary eclipse only $0.003 \mathrm{mag}$ deep is predicted in this scenario, but is undetectable in the photometry available. We conclude that while a planetary companion remains possible, a blend scenario is also consistent with all observational constraints and may be more likely given the significant crowding in the field toward the Galactic center. Further observations are needed to resolve the issue.

\subsection{OGLE-TR-33: a blend}

Our analysis of this star based on 4 Keck spectra shows very clearly that it is an example of a blend with an eclipsing binary (in this case, a triple system), with obvious asymmetries in the spectral lines that correlate with phase in the 1.95-day orbit. A full description of the observations and our results for OGLE-TR-33 will be reported elsewhere (Torres et al. 2003, in preparation). 


\subsection{OGLE-TR-56: a Jupiter-size companion}

The results from our radial velocities for this candidate were reported by Konacki et al. (2003), who showed that it is orbited by a planetary companion with a mass of $0.9 \pm 0.3 \mathrm{M}_{\text {Jup }}$ and a radius of $1.30 \pm 0.15 \mathrm{R}_{\mathrm{Jup}}$. The velocity measurements are listed in Table 2 . As indicated in that paper, the spectral line profiles show very little asymmetry and no discernible change with the phase in the orbit, supporting the reality of the velocity changes. The line bisectors for the individual spectra are shown in Figure 8c. Other than some contamination (at the level of 10\%) from scattered moonlight, which was removed using TODCOR as described by Konacki et al. (2003), we find no signs of another star in the spectra at the level of 3\% or more of the light of the primary.

The photometric period of the system, $P=1.21190$ days, appears stable over the oneyear interval spanned by the first and last recorded transits. The 12 individual transits are shown in Figure 13. Four of these transit events are complete, covering both the ingress and egress. The analysis of the out-of-eclipse variations by Sirko \& Paczyński (2003) does not indicate any significant ellipsoidal or heating effects, which would suggest a stellar companion.

Simulations as in the case of OGLE-TR-3 to test blend scenarios were carried out and reported by Konacki et al. (2003). However, it is important to note that in OGLE-TR-3 there is no significant velocity variation, which leaves open the possibility that contamination from a fainter eclipsing binary may be the underlying cause of the periodic drops in brightness. For OGLE-TR-56, on the other hand, the velocity changes are significant and cannot be explained by variable asymmetries in the line profiles. This makes the blend scenario very unlikely, and inconsistent with the available observations. We are conducting further spectroscopic and photometric observations of this system.

\subsection{OGLE-TR-58: planetary companion or blend}

A complication during the observation of this object arose at the telescope due to the presence of two relatively bright stars at separations of $1^{\prime \prime} .1$ and $1^{\prime \prime} .7$ on either side the target, more or less aligned with the spectrograph slit. This led us to modify the usual observing protocols regarding corrections for atmospheric dispersion. The image rotator routinely used with HIRES normally follows the parallactic angle so that atmospheric dispersion is oriented along the slit. For OGLE-TR-58 we set it at a different angle roughly $90^{\circ}$ away from nominal in order to avoid having the light from these two extra sources go down the slit along with that of the target. This introduces the risk of spurious velocity shifts whose magnitude will 
depend on the hour angle and elevation of the star at the time of the observation. Thus, the interpretation of the two radial velocity measurements we obtained for this candidate using the iodine cell (Table 3) is somewhat problematic. The formal difference is not significant, although as in the case of OGLE-TR-10 the trend with time is consistent with the phasing from the OGLE-III photometry, for the reported period of $P=4.34517$ days. The velocity amplitude based on the two available measurements is $K=200 \pm 94 \mathrm{~m} \mathrm{~s}^{-1}$, and the estimated mass of the companion is $1.6 \pm 0.8 \mathrm{M}_{\text {Jup }}$. The light curve analysis gives a tentative radius for the planet of $\sim 1.6 \mathrm{R}_{\mathrm{Jup}}$. Spectral line asymmetries are not large (Figure $8 \mathrm{~d}$ ).

We note, however, that the photometry is also problematic for this object, for several reasons: (1) The precision of the measurements is somewhat worse than for other stars of similar brightness in the OGLE-III survey, possibly due to the proximity of a bright star in the field that is heavily saturated (Udalski 2002, priv. comm.); (2) There appears to be a systematic offset in the light level outside of eclipse of about 0.02 mag between the 2001 season and the following season (see Figure 14), possibly related to the bright star mentioned above; (3) Only two clear dips in brightness have been recorded for this star, during the first of the OGLE-III seasons, with an interval of about 35 days. This renders the period rather uncertain. Although the reported period of 4.34 days fits best (including what appears to be a third incomplete transit during the 2002 season), other periods cannot be entirely ruled out with the existing data. Additional photometry is needed to resolve these issues.

Out-of-eclipse variations appear insignificant, according to the analysis by Sirko \& Paczyński (2003), although this result could be affected by the systematic offset noted above. From the data available we conclude that OGLE-TR-58 remains a good candidate for a transiting planet, although a blend scenario is equally possible.

\section{Final remarks}

Deep photometric searches for transiting extrasolar planets in the field (OGLE-III, Udalski et al. 2002a; EXPLORE, Mallén-Ornelas et al. 2003; and others) are beginning to uncover significant numbers of candidates among faint stars $(V=14-20)$. Similar searches in open clusters will soon follow suit (e.g., PISCES, Mochejska et al. 2002; STEPPS, Gaudi et al. 2002). Our spectroscopic follow-up of 5 of the OGLE-III candidates has presented us with a sampling of the results that may be expected from other deep surveys ${ }^{9}$, and has highlighted the challenges to be faced in confirming the planetary nature of any of these objects. After considering all the spectroscopic and photometric information together, two of our objects,

\footnotetext{
${ }^{9}$ Similar results have already been obtained recently by Yee et al. (2002), for the EXPLORE project.
} 
OGLE-TR-3 and OGLE-TR-33, show clear indications that the transit-like events are produced by contamination from an eclipsing binary along the same line of sight. On the other hand the case of OGLE-TR-56, reported earlier by Konacki et al. (2003), is unlikely to be a blend and is the only candidate with a measured radial velocity variation that is significant. It harbors a Jupiter-size companion in a very tight orbit with a period of only 1.2 days. OGLE-TR-10 and OGLE-TR-58 remain good candidates for having a planetary companion similar to Jupiter, but the data available so far also allow for the possibility that they are blends.

Our high-resolution spectroscopic observations of a number of standard stars have enabled us to characterize the stability of the HIRES instrument on Keck I quite accurately. We have shown that the main contribution to the errors of velocities determined by the classical Th-Ar technique appears to be systematics at the level of $\leq 100 \mathrm{~m} \mathrm{~s}^{-1}$, and is due to shifts of the spectrum on the detector beyond the observer's control. Numerical errors intrinsic to the technique are smaller $\left(\leq 50 \mathrm{~m} \mathrm{~s}^{-1}\right)$. Even for fainter stars such as the OGLE candidates, systematics dominate in most cases. This indicates that with proper care it is possible to measure Doppler shifts of faint stars to about $100 \mathrm{~m} \mathrm{~s}^{-1}$. We view this as a very encouraging result for future follow-up campaigns with this instrumentation. It is worth pointing out that, while precisions of a few $\mathrm{m} \mathrm{s}^{-1}$ may never be achievable for faint stars with current telescopes and instrumentation (and thus small planets in large orbits are beyond reach), a significant portion of parameter space remains in which variations of a few hundred $\mathrm{ms}^{-1}$ are still detectable in stars as faint as $V=17$, namely, planets similar to Jupiter with relatively short orbital periods of a few days. Precisions of $\sim 100 \mathrm{~m} \mathrm{~s}^{-1}$ such as we have demonstrated here are sufficient to detect such objects.

We have also shown that even better precision (and accuracy) of $50-60 \mathrm{~m} \mathrm{~s}^{-1}$ can be obtained for faint stars by using the iodine gas absorption cell on HIRES, with two modifications to the standard procedures that make the technique as efficient as the classical Th-Ar method. The first is that detailed PSF modeling is not required since the photon noise for faint stars is much larger than the level at which that modeling makes any difference. The second is that the modeling works sufficiently well with synthetic templates instead of observed templates, thus obviating the need to spend precious telescope time obtaining high $\mathrm{S} / \mathrm{N}$ spectra of each candidate without the iodine cell.

We are grateful to A. Udalski and the OGLE team for their many generous contributions to this project. We also thank K. Stanek for his continuous encouragement, and D. Mink for help with software issues. D.S. thanks S. Korzennik for his invaluable help in understanding precision radial velocities. The data presented herein were obtained at the W. M. Keck Observatory, which is operated as a scientific partnership among the California 
Institute of Technology, the University of California and the National Aeronautics and Space Administration. The Observatory was made possible by the generous financial support of the W. M. Keck Foundation. M.K. gratefully acknowledges the support of NASA through the Michelson fellowship program and partial support by the Polish Committee for Scientific Research, Grant No. 2P03D 001 22., and G.T. acknowledges support from NASA's Kepler mission. S.J. thanks the Miller Institute for Basic Research in Science at UC Berkeley for support through a research fellowship. This research has made use of the SIMBAD database, operated at CDS, Strasbourg, France, and of NASA's Astrophysics Data System Abstract Service.

\section{REFERENCES}

Barlow, T. 2002, MAKEE, MAuna Kea Echelle Extraction, http://spider.ipac.caltech.edu/staff/tab/makee/

Brown, T. M., Charbonneau, D., Gilliland, R. L., Noyes, R. W., \& Burrows, A. 2001, ApJ, 552,699

Butler, R. P., Marcy, G. W., Williams, E., McCarthy, C., Dosanjh, P., \& Vogt, S. S. 1996, PASP, 108, 500

Charbonneau, D., Brown, T. M., Latham, D. W., \& Mayor, M. 2000, ApJ, 529, L45

Charbonneau, D. 2003, in Scientific Frontiers in Research on Extrasolar Planets, eds. D. Deming \& S. Seager (San Francisco: ASP), ASP Conf. Ser., 294, 449

Charbonneau, D., Brown, T. M., Noyes, R. W., \& Gilliland, R. L. 2002, ApJ, 568, 377

Cody, A. M., \& Sasselov, D. D. 2002, ApJ, 569, 451

Drake, A. J. 2003, ApJ, 589, 1020

Dreizler, S., Hauschildt, P. H., Kley, W., Rauch, T., Schuh, S. L., Werner, K., \& Wolff, B. 2003, A\&A, 402, 791

Dreizler, S., Rauch, T., Hauschildt, P., Schuh, S. L., Kley, W., \& Werner, K. 2002, A\&A, 391, L17

Fischer, D. A., \& Valenti, J. 2003, in Scientific Frontiers in Research on Extrasolar Planets, eds. D. Deming \& S. Seager (San Francisco: ASP), ASP Conf. Ser., 294, 117 
Gaudi, B. S., Burke, C. J., DePoy, D. L., Marshall, J. L., Pogge, R. W., \& STEPSS Collaboration, 2002, BAAS, 34, 1264

Gray, D. F. 1992, The Observation and Analysis of Stellar Photospheres (2nd Ed.; Cambridge: Cambridge Univ. Press, 417

Henry, G. W., Marcy, G. W., Butler, R. P., \& Vogt, S. S. 2000, ApJ, 529, L4

Horne, K. 2003, in Scientific Frontiers in Research on Extrasolar Planets, eds. D. Deming \& S. Seager (San Francisco: ASP), ASP Conf. Ser., 294, 361

Konacki, M. \& Wolszczan, A. 2003, ApJ, to appear, astro-ph/0305536

Konacki, M., Torres, G., Jha, S., \& Sasselov, D. D. 2003, Nature, 421, 507

Korzennik, S. G., Brown, T. M., Fischer, D. A., Nisenson, P., \& Noyes, R. W. 2000, ApJ, 533, L147

Kurtz, M. J., \& Mink, D. J. 1998, PASP, 110, 934

Kurucz, R. L. 1995, ASP Conf. Ser. 78: Astrophysical Applications of Powerful New Databases, 205

Latham, D. W. 2003, in Scientific Frontiers in Research on Extrasolar Planets, eds. D. Deming \& S. Seager (San Francisco: ASP), ASP Conf. Ser., 294, 409

Mallén-Ornelas, G., Seager, S., Yee, H. K. C., Minniti, D., Gladders, M. D., Mallén-Fullerton, G. M., \& Brown, T. M. 2003, ApJ, 582, 1123

Marcy, G. W., Cochran, W. D., \& Mayor, M. 2000, Protostars and Planets IV, 1285

Mazeh, T., Naef, D., Torres, G., Latham, D. W., Mayor, M., Beuzit, J., Brown, T. M., Buchhave, L., Burnet, M., Carney, B. W., Charbonneau, D., Drukier, G. A., Laird, J. B., Pepe, F., Perrier, C., Queloz, D., Santos, N. C., Sivan, J., Udry, S., \& Zucker, S. 2000, ApJ, 532, L55

Mochejska, B. J., Stanek, K. Z., Sasselov, D. D., \& Szentgyorgyi, A. H. 2002, AJ, 123, 3460

Queloz, D., Henry, G. W., Sivan, J. P., Baliunas, S. L., Beuzit, J. L., Donahue, R. A., Mayor, M., Naef, D., Perrier, C., \& Udry, S. 2001, A\&A, 379, 279

Sasselov, D. D. 2003, ApJ, in press, astro-ph/0303403

Schneider, J. 2003, The Extrasolar Planets Encyclopaedia, http://www. obspm.fr/encycl/encycl.html 
Seager, S. \& Mallén-Ornelas, G. 2003, ApJ, 585, 1038

Sirko, E., \& Paczyński, B. 2003, astro-ph/0302175

Tinney, C. G., Butler, R. P., Marcy, G. W., Jones, H. R. A., Penny, A. J., Vogt, S. S., Apps, K., \& Henry, G. W. 2001, ApJ, 551, 507

Udalski, A., Szewczyk, O., Żebruń, K., Pietrzyński, G., Szymański, M., Kubiak, M., Soszyński, I., \& Wyrzykowski, Ł. 2002c, Acta Astronomica, 52, 317

Udalski, A., Paczyński, B., Żebruń, K., Szymański, M., Kubiak, M., Soszyński, I., Szewczyk, O., Wyrzykowski, Ł., \& Pietrzyński, G. 2002a, Acta Astronomica, 52, 1

Udalski, A., Pietrzyński, G., Szymański, M., Kubiak, M., Żebruń, K., Soszyński, I., Szewczyk, O., \& Wyrzykowski, Ł. 2003, astro-ph/0306444

Udalski, A., Żebruń, K., Szymański, M., Kubiak, M., Soszyński, I., Szewczyk, O., Wyrzykowski, Ł., \& Pietrzyński, G. 2002b, Acta Astronomica, 52, 115

Udry, S., Mayor, M., Naef, D., Pepe, F., Queloz, D., Santos, N. C., Burnet, M., Confino, B., \& Melo, C. 2000, A\&A, 356, 590

Vidal-Madjar, A., des Etangs, A. L., Désert, J.-M., Ballester, G. E., Ferlet, R., Hébrard, G., \& Mayor, M. 2003, Nature, 422, 143

Vogt, S. S. et al. 1994, Proc. SPIE, 2198, 362

Vogt, S. S., Marcy, G. W., Butler, R. P., \& Apps, K. 2000, ApJ, 536, 902

Wolszczan, A., \& Frail, D. A. 1992, Nature, 355, 145

Yee, H. K. C., Mallen-Ornelas, G., Seager, S., Gladders, M. D., Brown, T., Minniti, D., Ellison, S., \& Mallen-Fullerton, G. 2002, astro-ph/0208355

Zucker, S., \& Mazeh, T. 1994, ApJ, 420, 806 
Fig. 1. - Portion of the observed (co-added) spectra around the $\mathrm{H} \beta$ line for four of our OGLE candidates, with the best-fit synthetic spectra superimposed (smooth solid line). The derived stellar parameters are indicated.

Fig. 2.- Record of the shifts (in pixels) measured on the HIRES detector as a function of time during our Keck run. Filled circles represent the shifts of each of the Th-Ar exposures compared to the first such frame, and open symbols correspond to shifts measured on stellar exposures of standard stars and $\mathrm{K}$ stars with the iodine cell, also compared to the first such exposure (see text). An offset has been applied to the iodine shifts to bring them into agreement with the Th-Ar shifts. Error bars for each shift are plotted, but are smaller than the symbol sizes. Vertical dotted lines show the times of the exposures for the OGLE candidates.

Fig. 3.- Close-up of Figure 2 showing the shifts as measured from the Th-Ar exposures separately for each night. The times of each OGLE observation are shown with vertical dotted lines, and labeled with the number of the candidate (e.g., '33' for OGLE-TR-33). Circled numbers indicate iodine exposures.

Fig. 4.- Differential radial velocities of the five 'constant' K dwarfs HIP 1078, HIP 2102, HIP 3232, HIP 117177, and HIP 117846 determined with the Th-Ar technique, and referred to the velocities of HIP 1334. Systematic effects have been removed using the iodine (see text), and the average for each star has been subtracted. The standard deviation of these differences is $49 \mathrm{~ms}^{-1}$.

Fig. 5.- Differential radial velocities of HD 179949 (a) and HD 209458 (b) relative to HIP 1334, determined with the Th-Ar technique and corrected for systematics (see text). The solid lines represent the orbits due to the known planetary companions of these stars (Mazeh et al. 2000; Tinney et al. 2001). Residuals of the Th-Ar velocities from these orbits give standard deviations of $41 \mathrm{~m} \mathrm{~s}^{-1}$ for HD 179949 (c) and $49 \mathrm{~m} \mathrm{~s}^{-1}$ for HD 209458 (d).

Fig. 6. - Differential radial velocities (HD 209458 minus HD 179949) from the Th-Ar technique, corrected for instrumental shifts using the iodine orders (a). This is analogous to Figure 5b, except for the use of HD 179949 as star 1 instead of HIP 1334 (see text). The differential orbit computed from the elements published by Mazeh et al. (2000) and Tinney et al. (2001) are indicated by the solid line. The shaded area represents the uncertainty introduced by errors in the orbital elements. After subtracting the orbital variations, the standard deviation of the differential Th-Ar velocities is $69 \mathrm{~m} \mathrm{~s}^{-1}$ (b).

Fig. 7. - The radial velocities of HD 179949 (a) and HD 209458 (b) determined with the $I_{2}$ cell using synthetic templates. The solid line represents the calculated velocity differences 
(Mazeh et al. 2000; Tinney et al. 2001). After subtracting the orbital variations, the standard deviation of the $I_{2}$ velocities is $22 \mathrm{~m} \mathrm{~s}^{-1}$ for HD 179949 (c) and $26 \mathrm{~m} \mathrm{~s}^{-1}$ for HD 209458 (d). As a test, we derived the iodine velocities also using observed templates (open circles). The scatter in this case is smaller (see text).

Fig. 8. - Spectral line bisectors for OGLE-TR-3, OGLE-TR-10, OGLE-TR-56 (excluding a weak exposure), and OGLE-TR-58, labeled with the date (MJD) of each observation. There are no asymmetries significantly larger than in the Sun (see text), or any correlations with photometric phase.

Fig. 9.- (a) Radial velocity measurements of OGLE-TR-3 by Dreizler et al. (2003) along with our orbital fit of those data, adopting the ephemeris in eq. 3. The velocity amplitude is insignificant: $K=0.100 \pm 0.061 \mathrm{~km} \mathrm{~s}^{-1}$; (b) OGLE photometry phased with the same ephemeris. The hint of a secondary eclipse at phase 0.5 and the out-of-eclipse variations are very suggestive (see text).

Fig. 10. - Light curve fit resulting from a blend model with a grazing eclipsing binary in the background of OGLE-TR-3. The physical properties of the latter star (effective temperature, rotational velocity, surface gravity) are constrained by the estimates from our Keck spectra. The eclipsing binary in this model consists of a mid-K star orbiting a mid-F star, with an inclination angle of $82^{\circ}$. (a) Full light curve; (b) Enlargement of primary eclipse.

Fig. 11.- Individual transits of OGLE-TR-10 recorded in the OGLE-III photometry during the 2001 and 2002 observing seasons. Julian dates for the corresponding night are indicate for each panel. A tentative fitted model is shown for reference.

Fig. 12.- Light curve fit resulting from a blend model with an edge-on eclipsing binary in the background of OGLE-TR-10. The physical properties of the latter star (effective temperature, rotational velocity, surface gravity) are constrained by the estimates from our Keck spectra. The eclipsing binary in this model consists of an early-type $\mathrm{M}$ star orbiting a G0 star. (a) Full light curve; (b) Enlargement of primary eclipse.

Fig. 13.- Individual transits of OGLE-TR-56 recorded in the OGLE-III photometry during the 2001 and 2002 observing seasons. Julian dates for the corresponding night are indicate for each panel. The fitted model from Konacki et al. (2003) is shown for reference.

Fig. 14.- OGLE-III photometry for the candidate OGLE-TR-58, as a function of time. A systematic offset of about 0.02 mag between the two observing seasons is apparent, and is possibly related to the presence of a bright saturated star nearby. 

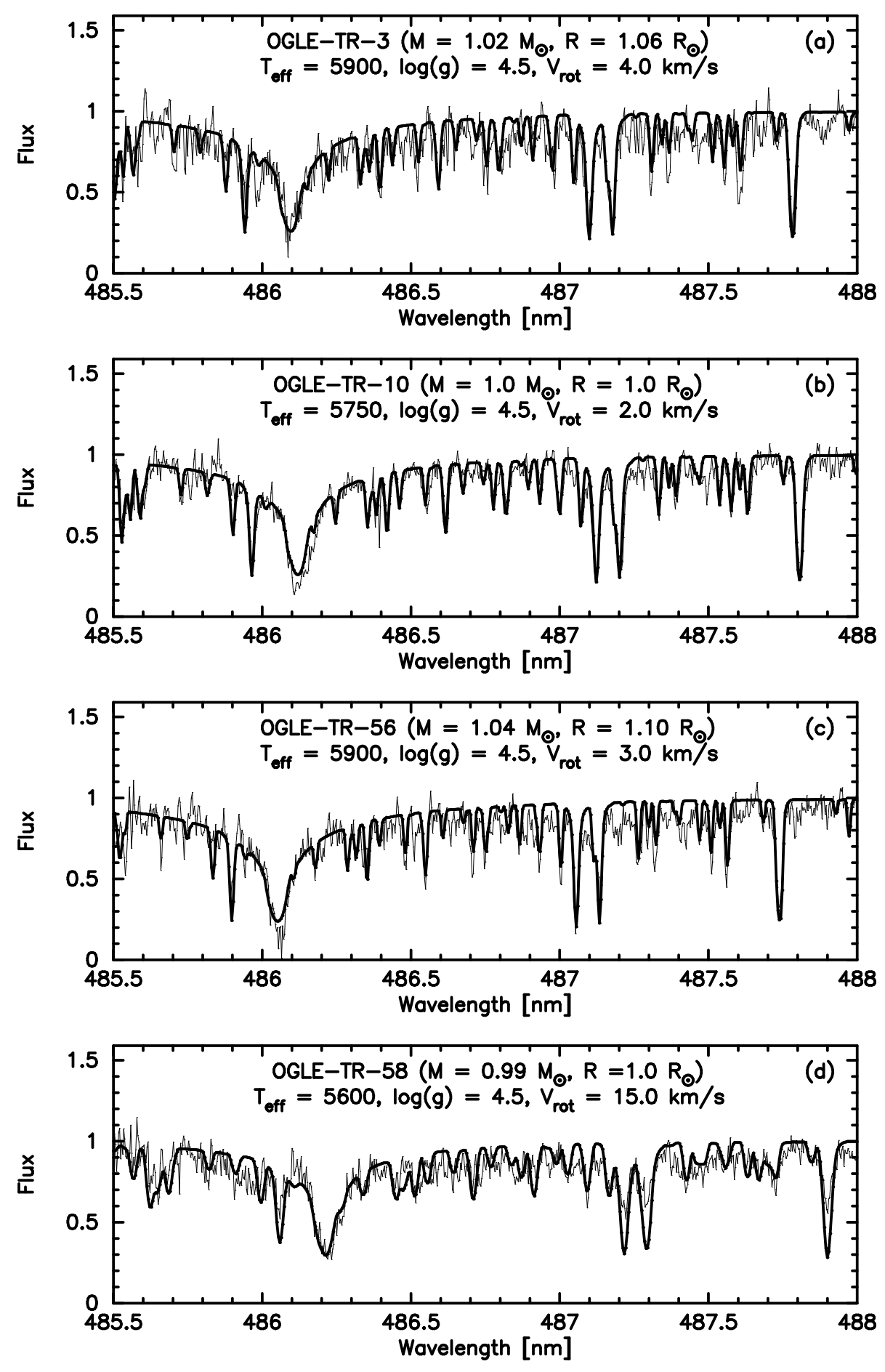

Fig. 1.- 


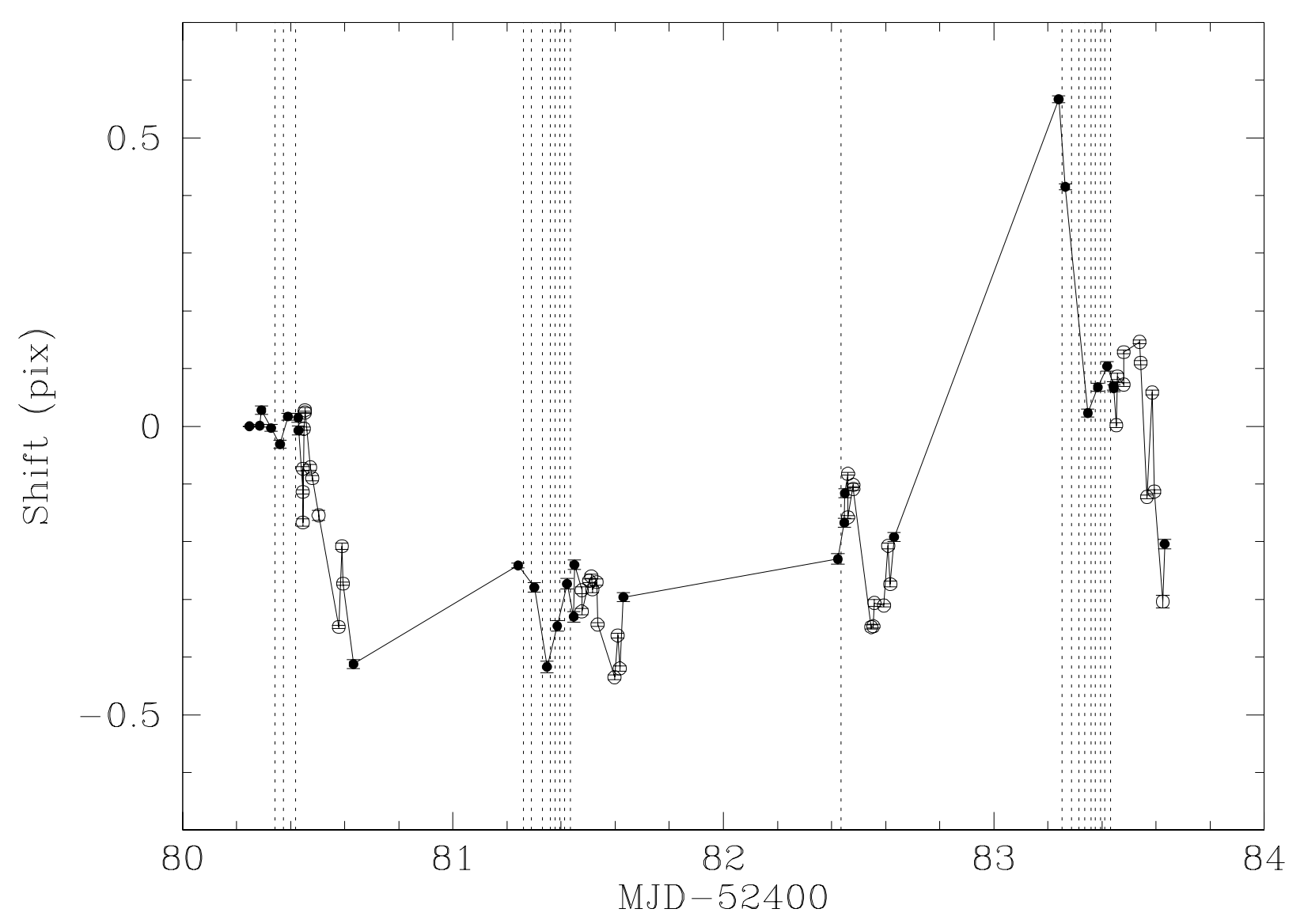

Fig. 2.- 

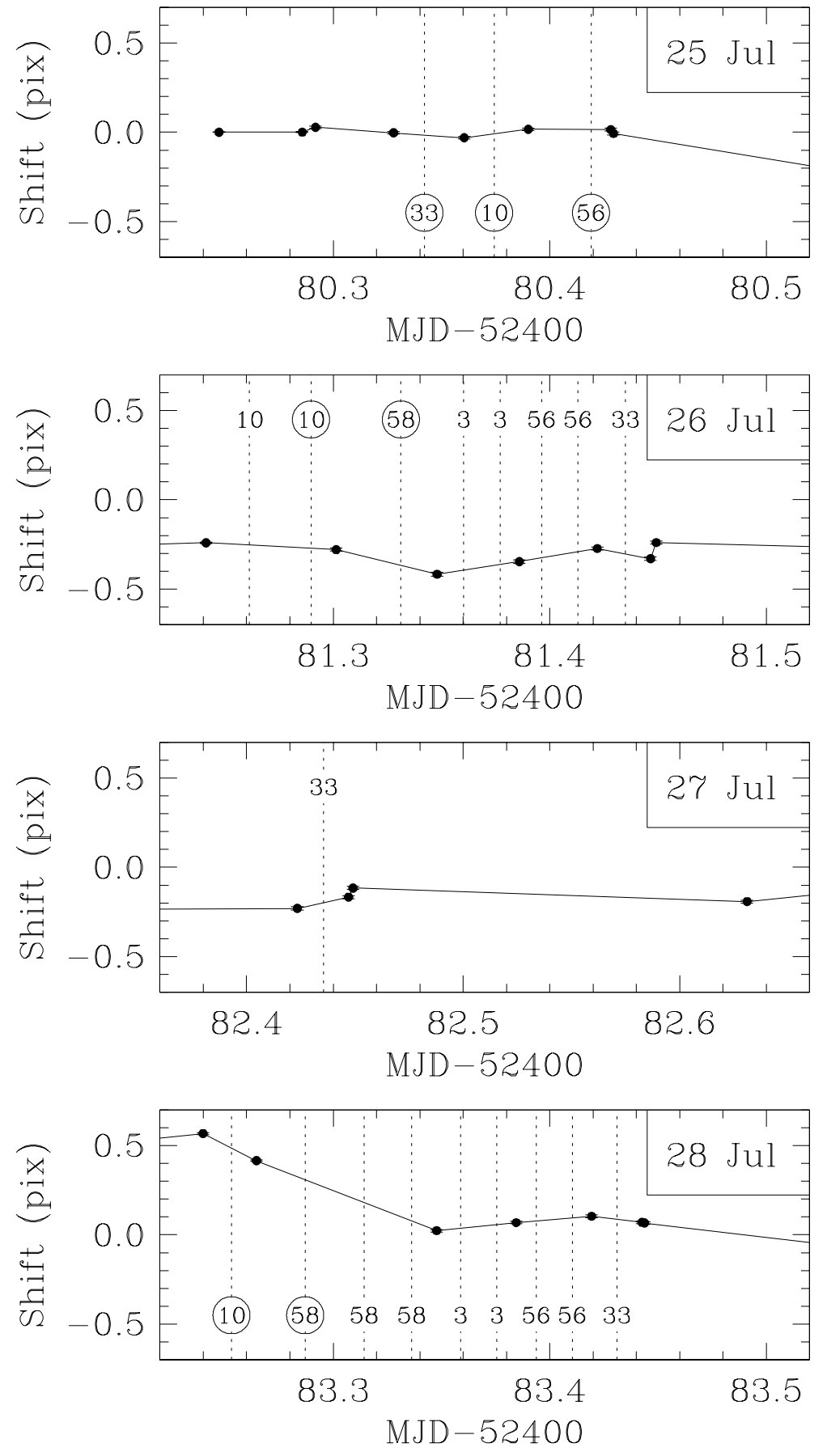

Fig. 3.- 


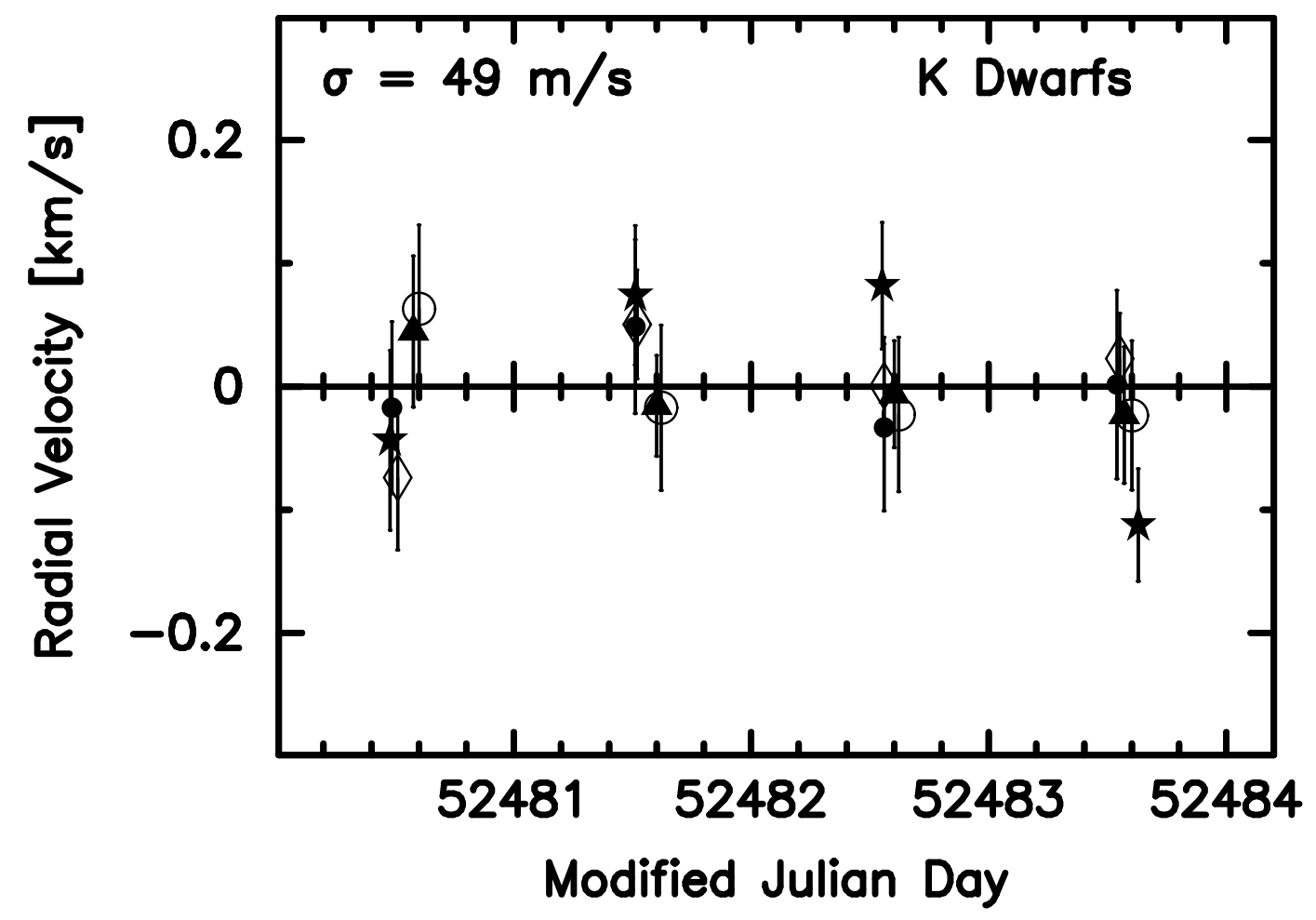

Fig. 4.- 

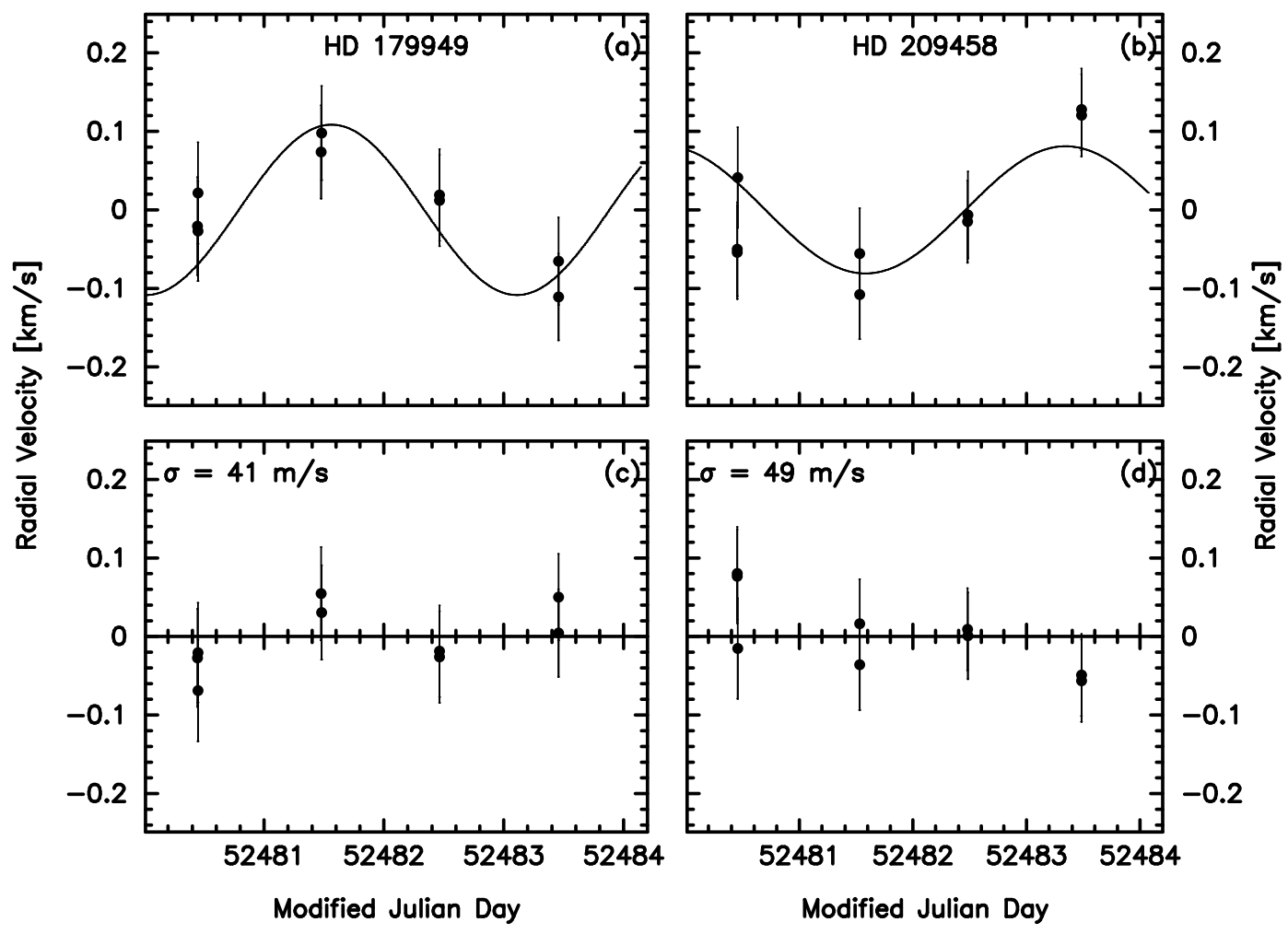

Fig. 5.- 


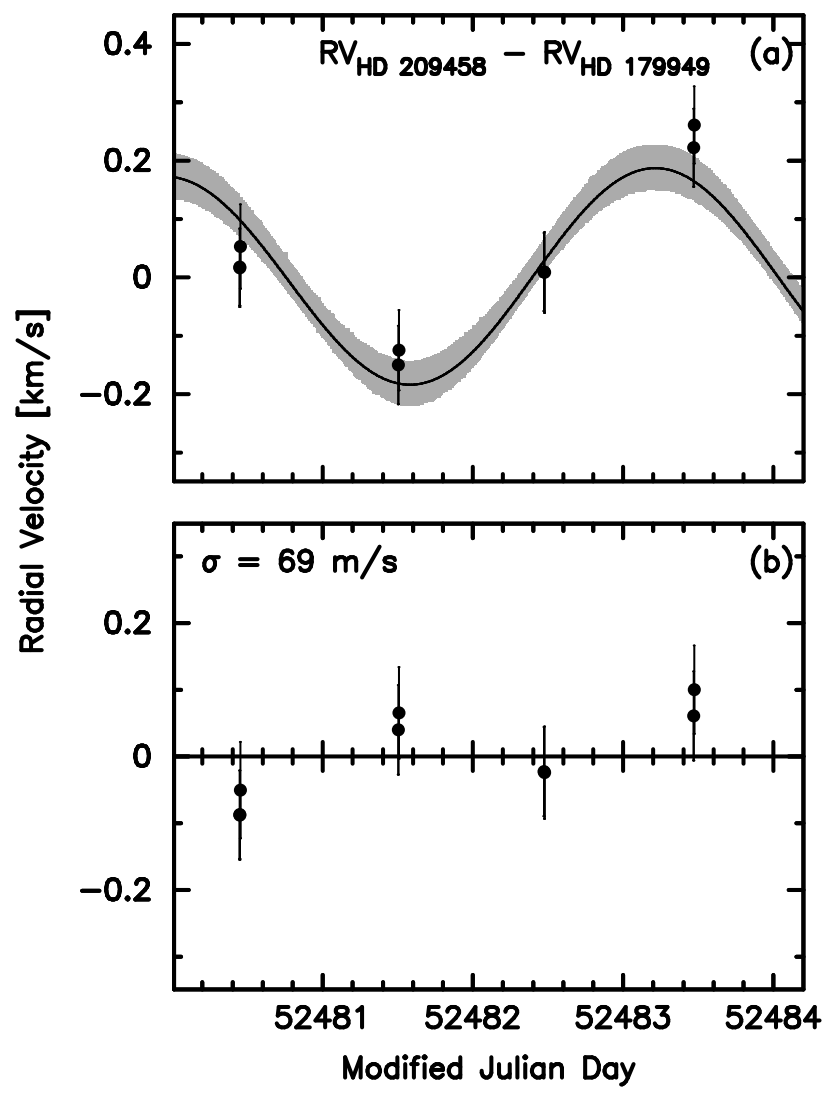

Fig. 6.- 

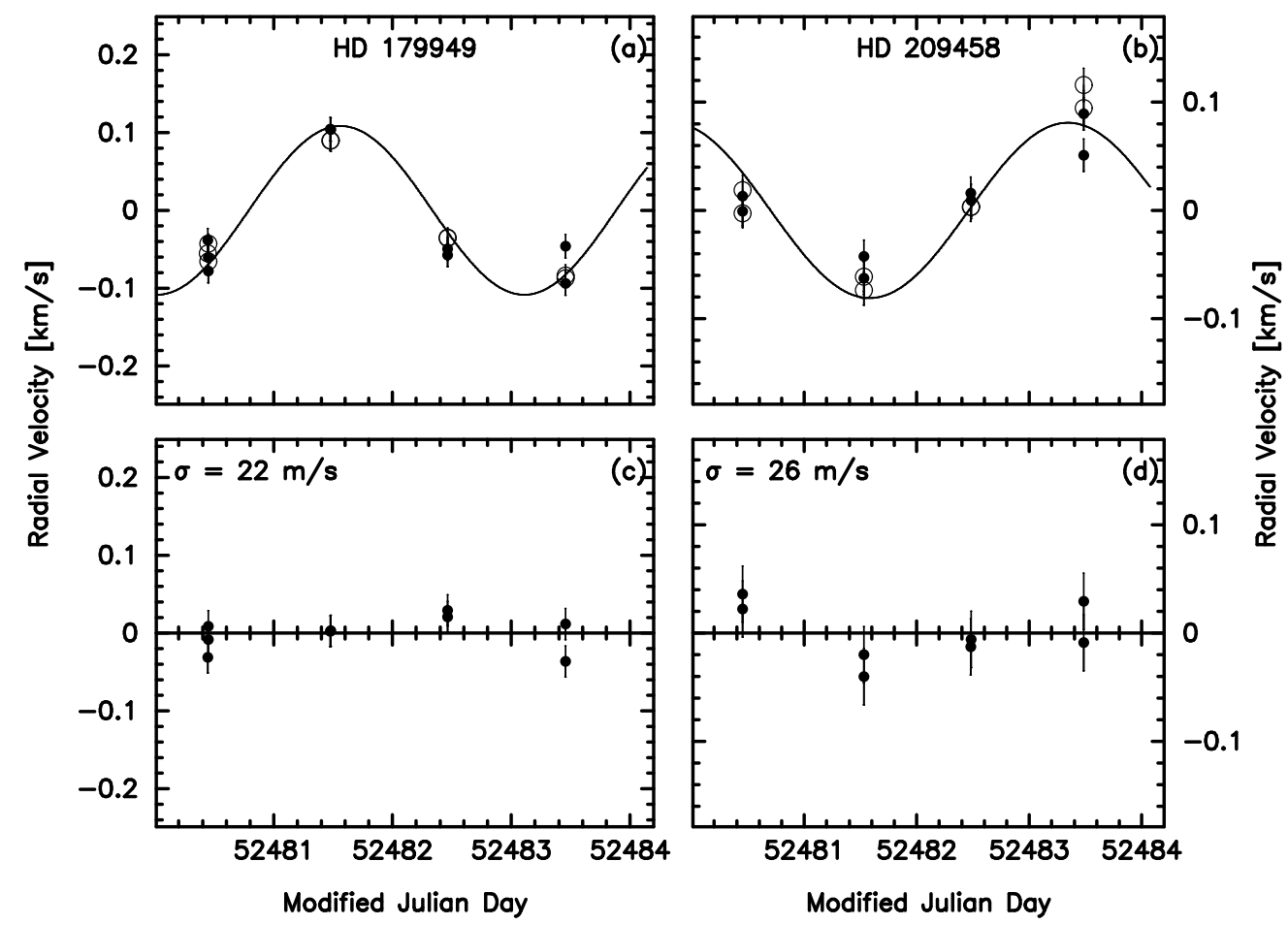

Fig. 7.- 

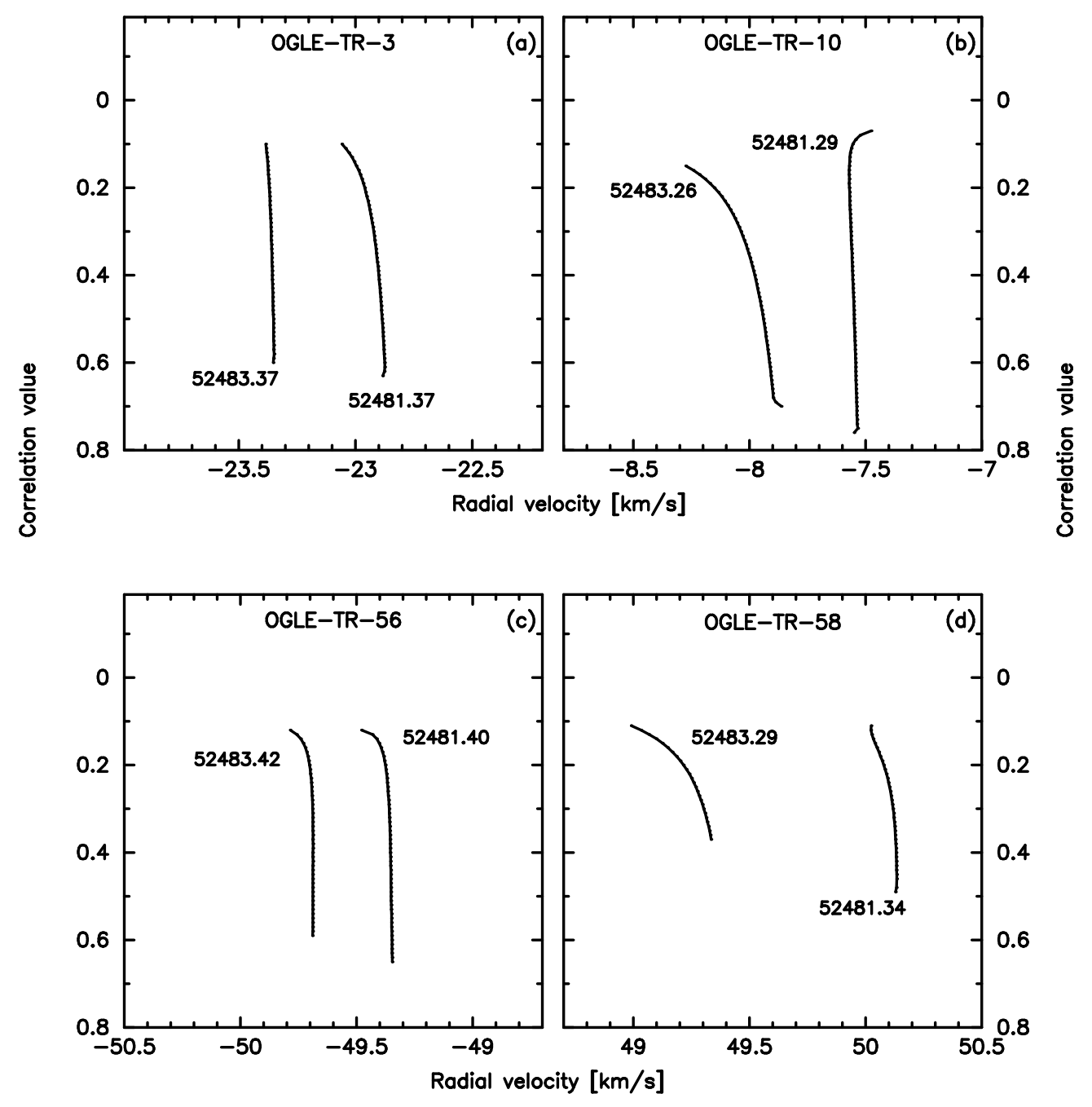

Fig. 8.- 

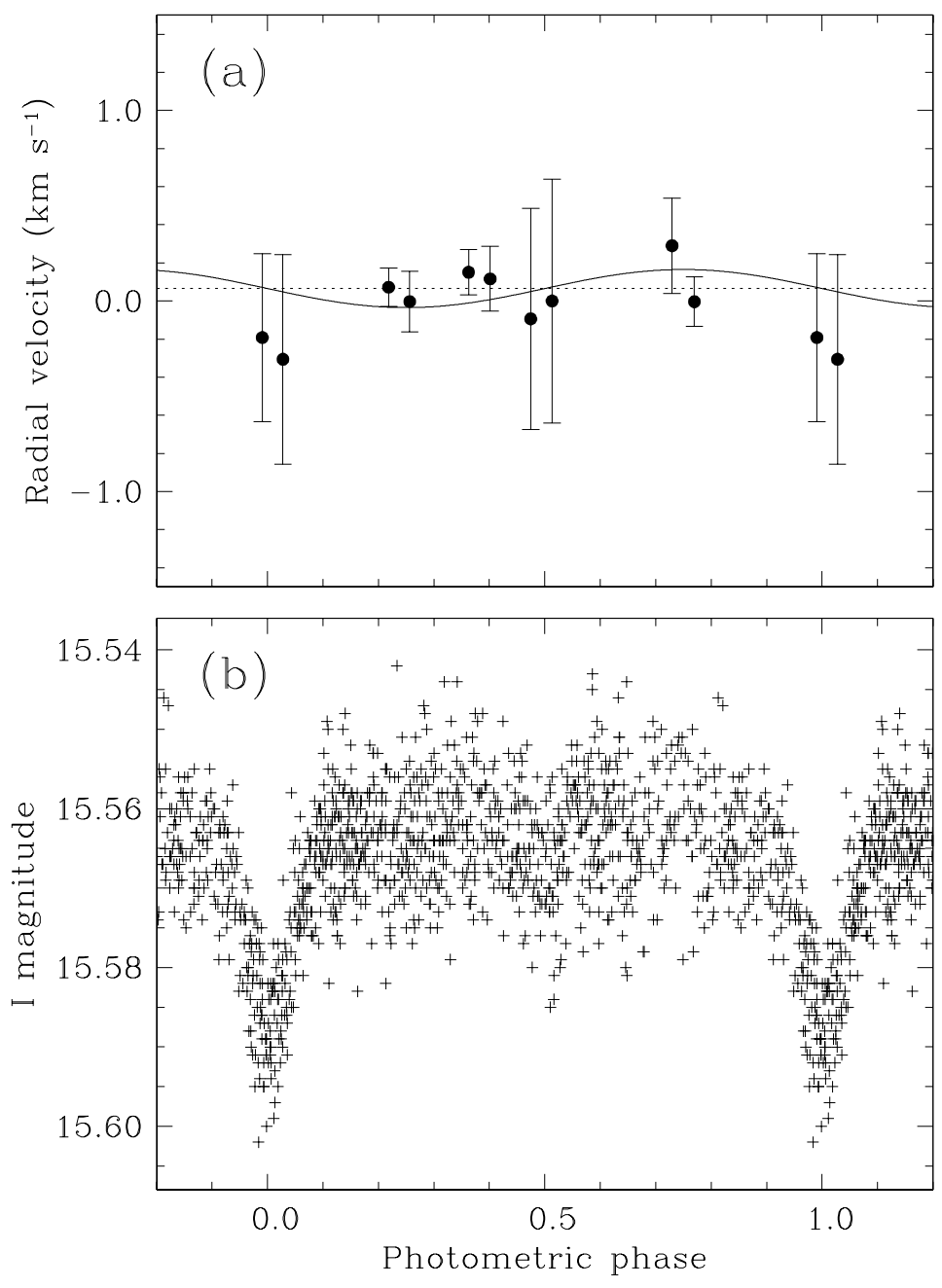

Fig. 9.- 

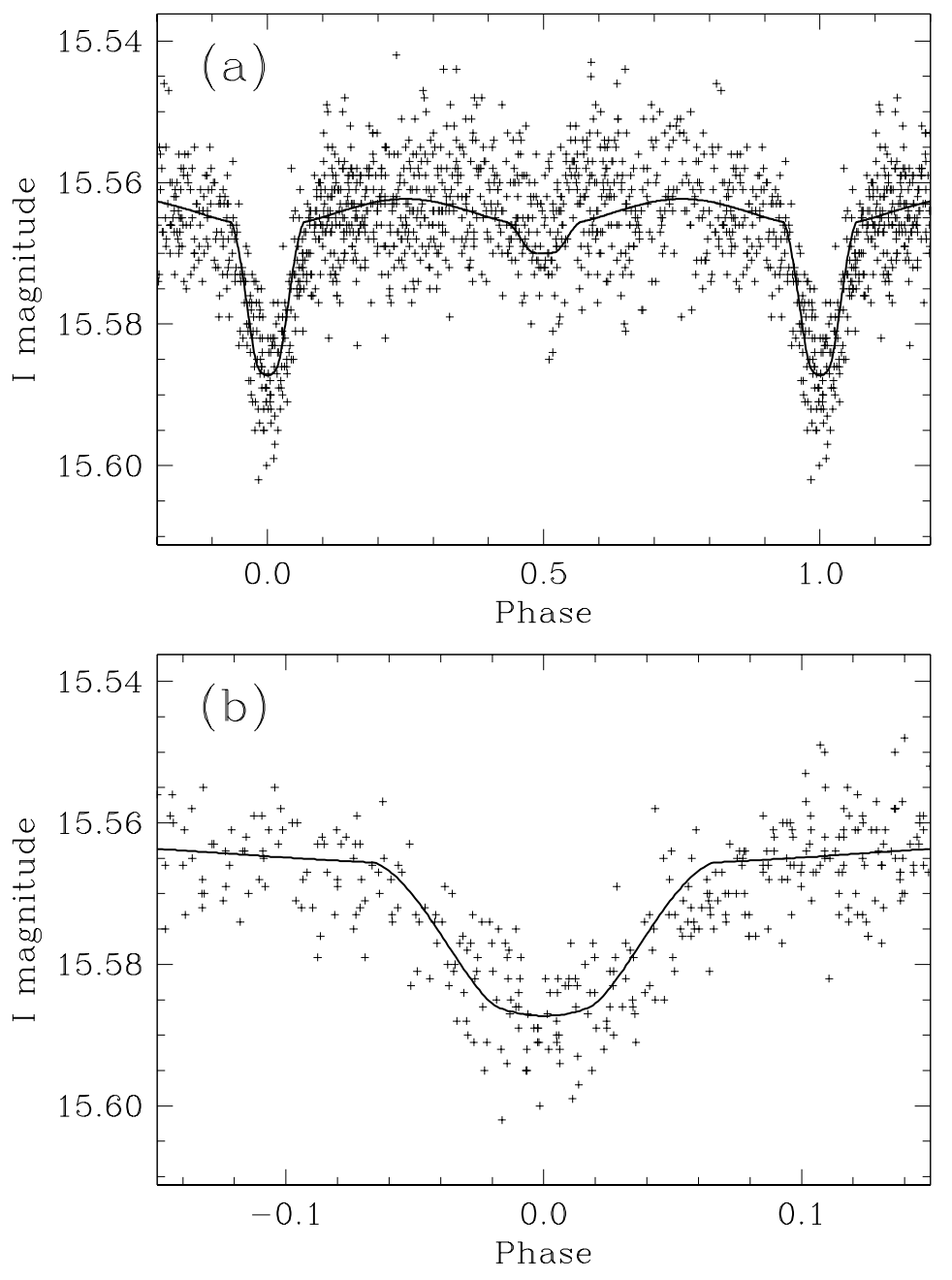

Fig. 10.- 


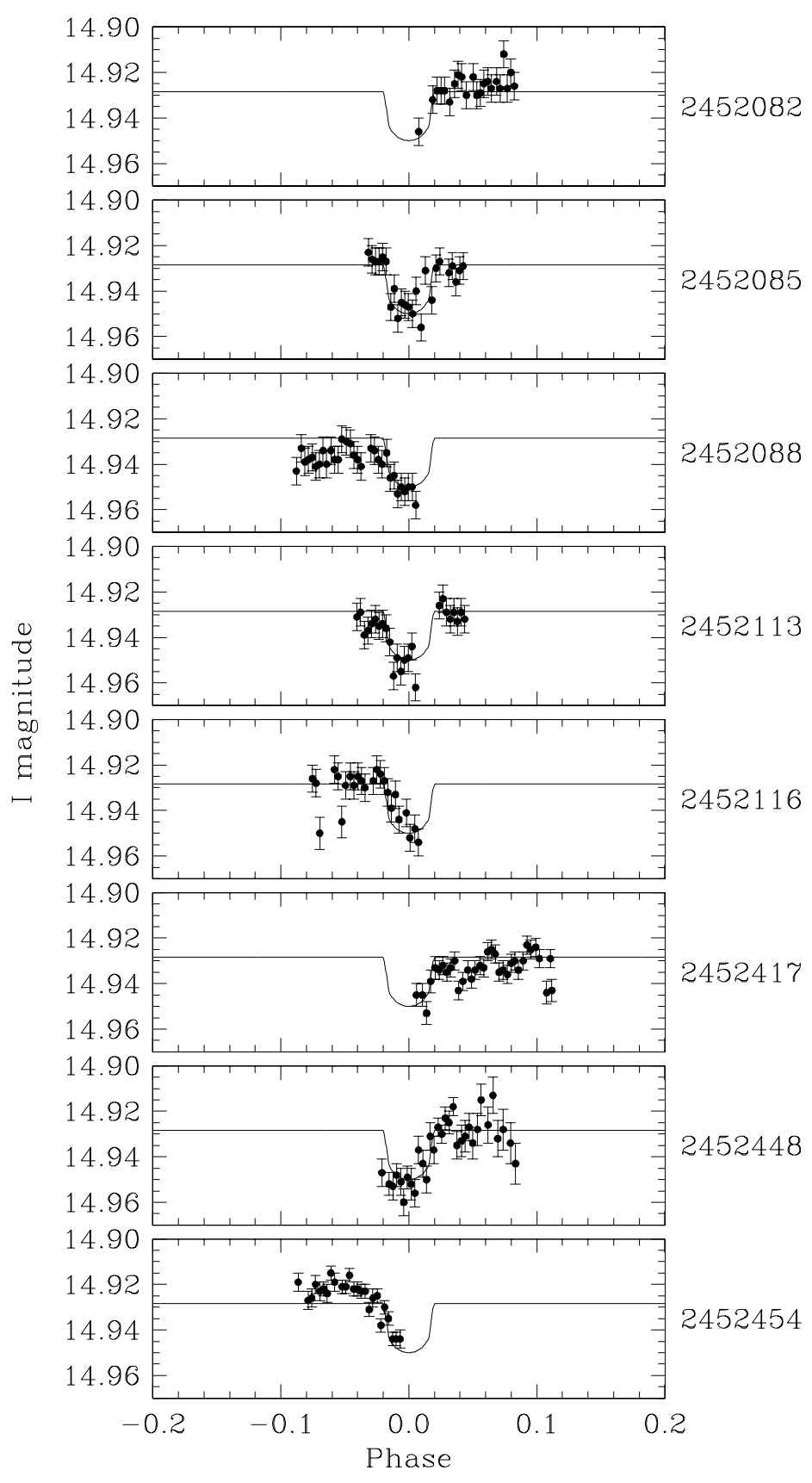

Fig. 11.- 

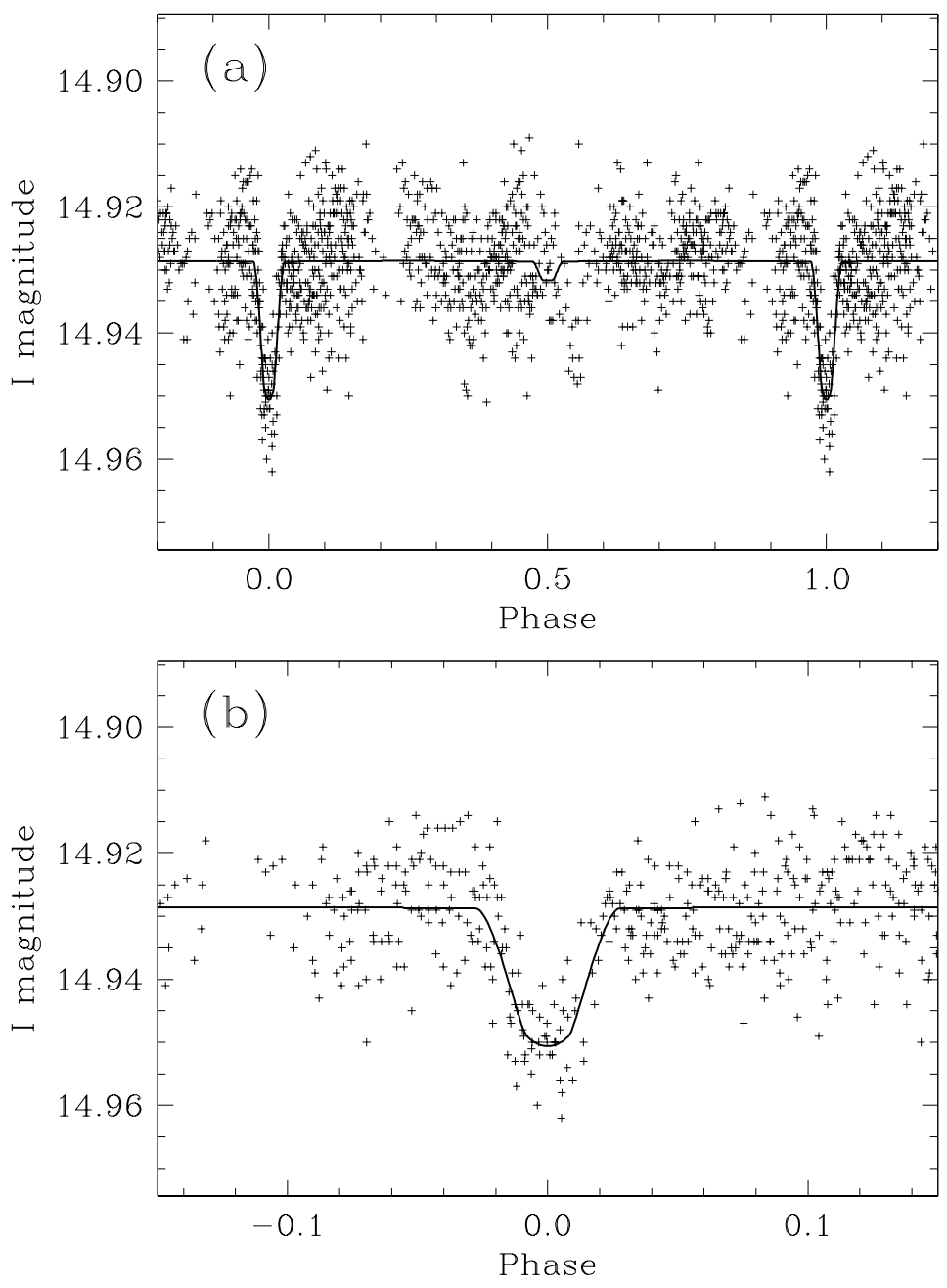

Fig. 12.- 


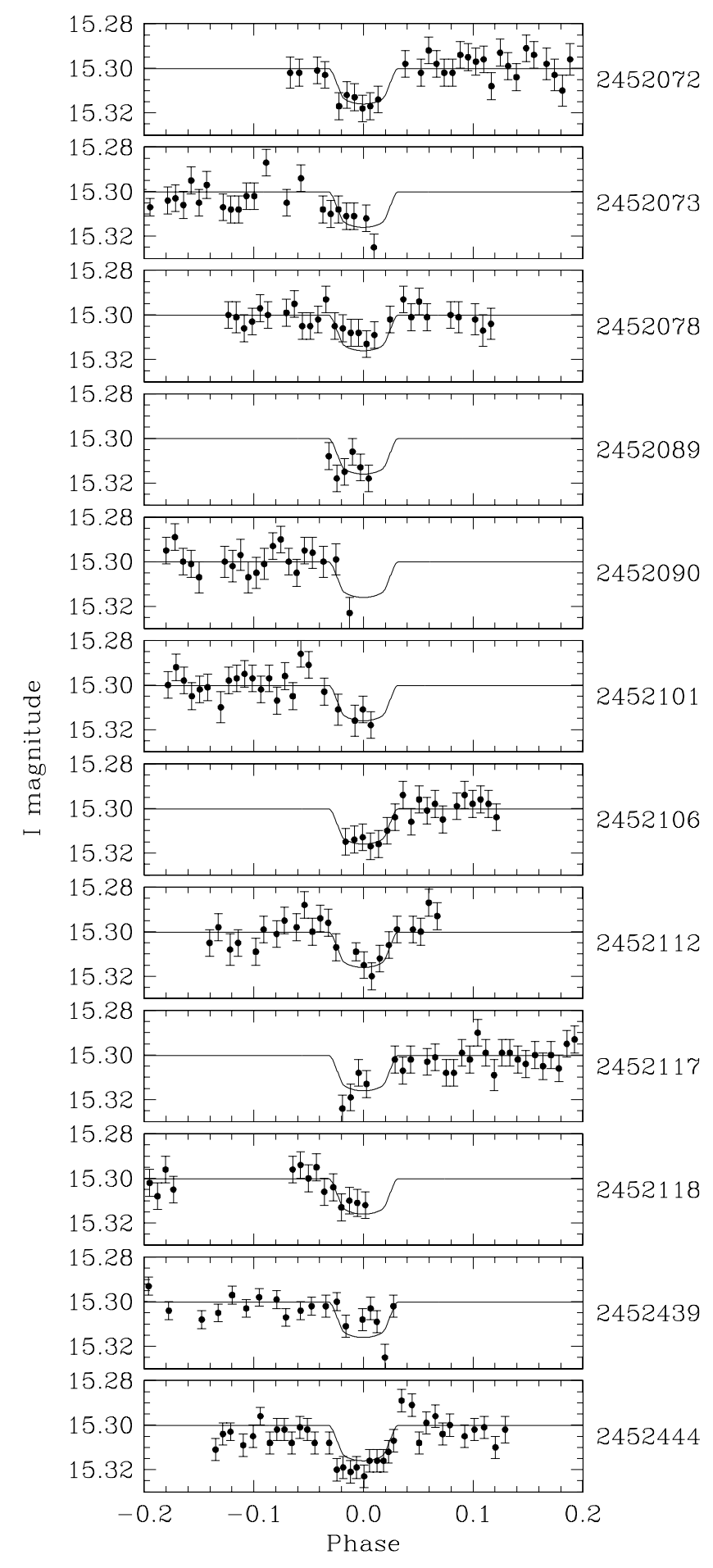

Fig. 13.- 


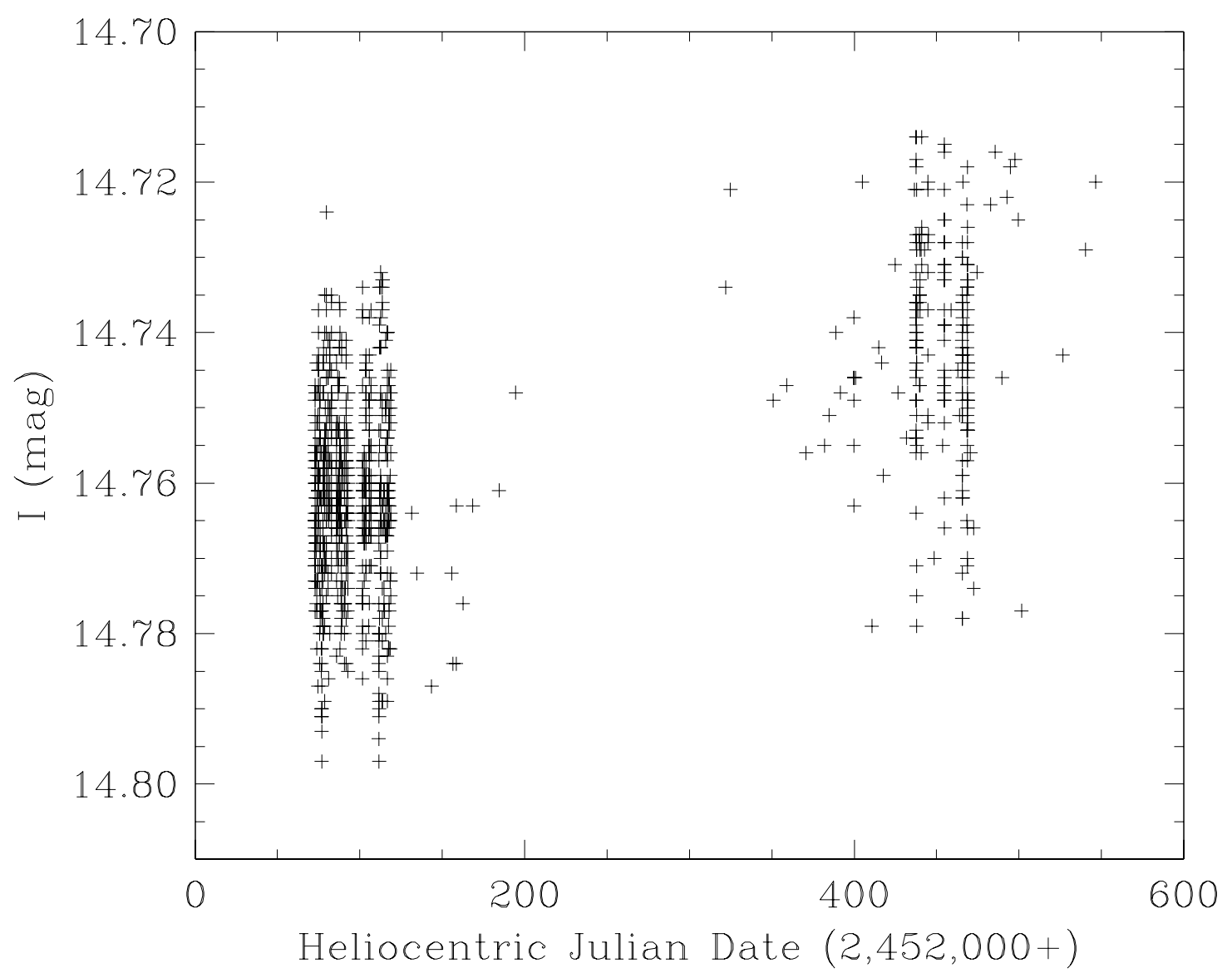

Fig. 14.- 
Table 1. Program Stars.

\begin{tabular}{lcccc}
\hline \hline \multicolumn{1}{c}{ Star } & R.A. (J2000) & Dec. $(\mathrm{J} 2000)$ & $V$ (mag) & Exp. time (sec) \\
\hline OGLE-TR-3 & $17^{h} 51^{m} 48^{s} .95$ & $-30^{\circ} 13^{\prime} 25^{\prime \prime} .1$ & 16.6 & 1350 \\
OGLE-TR-10 & $17^{h} 51^{m} 28^{s} .25$ & $-29^{\circ} 52^{\prime} 34^{\prime \prime} .9$ & 15.8 & $1800,2700^{\mathrm{a}}$ \\
OGLE-TR-56 & $17^{h} 56^{m} 35^{s} .51$ & $-29^{\circ} 32^{\prime} 21^{\prime \prime} .2$ & 16.6 & 1350 \\
OGLE-TR-58 & $17^{h} 55^{m} 08^{s} .27$ & $-29^{\circ} 48^{\prime} 51^{\prime \prime} .3$ & 16.0 & $2700,2 \times 1800^{\mathrm{a}}$ \\
HD 179949 & $19^{h} 15^{m} 33^{s} .23$ & $-24^{\circ} 10^{\prime} 45^{\prime \prime} .7$ & 6.3 & $60,60^{\mathrm{a}}$ \\
HD 209458 & $22^{h} 03^{m} 10^{s} 80$ & $+18^{\circ} 53^{\prime} 04^{\prime \prime} .0$ & 7.7 & $60,60^{\mathrm{a}}$ \\
HIP 1078 & $00^{h} 13^{m} 24^{s} .64$ & $+19^{\circ} 04^{\prime} 16^{\prime \prime} .8$ & 9.9 & $300,600^{\mathrm{a}}$ \\
HIP 1334 & $00^{h} 16^{m} 43^{s} .37$ & $+20^{\circ} 51^{\prime} 24^{\prime \prime} .1$ & 10.0 & $300,600^{\mathrm{a}}$ \\
HIP 2102 & $00^{h} 26^{m} 40^{s} .48$ & $+30^{\circ} 11^{\prime} 58^{\prime \prime} .0$ & 10.1 & $300,600^{\mathrm{a}}$ \\
HIP 3232 & $00^{h} 41^{m} 07^{s} .90$ & $+19^{\circ} 15^{\prime} 15^{\prime \prime} .9$ & 9.9 & $300,600^{\mathrm{a}}$ \\
HIP 117177 & $23^{h} 45^{m} 24^{s} .64$ & $+39^{\circ} 07^{\prime} 24^{\prime \prime} .2$ & 10.2 & $300,600^{\mathrm{a}}$ \\
HIP 117846 & $23^{h} 54^{m} 04^{s} .83$ & $+17^{\circ} 33^{\prime} 00^{\prime \prime} .2$ & 10.1 & $300,600^{\mathrm{a}}$ \\
\hline
\end{tabular}

aTemplate exposure for use in the iodine reductions. 
Table 2. Thorium-Argon radial velocities in the barycentric frame for the OGLE targets.

\begin{tabular}{ccc}
\hline \hline HJD & Velocity $\left(\mathrm{km} \mathrm{s}^{-1}\right)$ & Error $\left(\mathrm{km} \mathrm{s}^{-1}\right)$ \\
\hline & OGLE-TR-3 \\
\hline 2452481.87318 & -23.03 & 0.35 \\
2452483.87185 & -23.40 & 0.07 \\
\hline \multicolumn{3}{c}{ OGLE-TR-56 } \\
\hline 2452480.92390 & -49.26 \\
2452481.90961 & -49.35 & 0.20 \\
2452483.90680 & -49.64 & 0.07 \\
\hline
\end{tabular}


Table 3. Iodine radial velocities for the OGLE targets. $^{\mathrm{a}}$

\begin{tabular}{ccc}
\hline \hline HJD & Velocity $\left(\mathrm{km} \mathrm{s}^{-1}\right)$ & Error $\left(\mathrm{km} \mathrm{s}^{-1}\right)$ \\
\hline \multicolumn{3}{c}{ OGLE-TR-10 } \\
\hline 2452481.79455 & +0.09 & 0.06 \\
2452483.75773 & -0.09 & 0.05 \\
\hline \multicolumn{3}{c}{ OGLE-TR-58 } \\
\hline 2452481.83607 & -0.18 \\
2452483.79181 & +0.18 & 0.16 \\
\hline
\end{tabular}

aThese velocities are referred to the average for each star, since the iodine technique yields only relative velocities. 